\title{
Regular $F$-manifolds: initial conditions and Frobenius metrics
}

\author{
Liana David and Claus Hertling
}

\begin{abstract}
A regular $F$-manifold is an $F$-manifold (with Euler field) $(M, \circ, e, E)$, such that the endomorphism $\mathcal{U}(X):=E \circ X$ of $T M$ is regular at any $p \in M$. We prove that the germ $((M, p), \circ, e, E)$ is uniquely determined (up to isomorphism) by the conjugacy class of $\mathcal{U}_{p}: T_{p} M \rightarrow T_{p} M$. We obtain that any regular $F$-manifold admits a preferred system of local coordinates and we find conditions, in these coordinates, for a metric to be Frobenius. We study the Lie algebra of infinitesimal symmetries of regular $F$-manifolds. We show that any regular $F$-manifold is locally isomorphic to the parameter space of a Malgrange universal connection. We prove an initial condition theorem for Frobenius metrics on regular $F$-manifolds.
\end{abstract}

Key words: (regular) F-manifolds, Frobenius metrics, canonical coordinates, generalized Darboux-Egoroff equations, infinitesimal symmetries, meromorphic connections.

2010 MS Classification: 32B10, 32G99, 53Z05, 53D45.

\section{Introduction}

Frobenius manifolds were defined in [2], by Boris Dubrovin, as a geometrization of the so called Witten-Dijkgraaf-Verlinde-Verlinde (WDVV)-equations and appear in many areas of mathematics (quantum cohomology, singularity theory, integrable systems etc). Later on, the weaker notion of $F$-manifold was introduced in the literature by Hertling and Manin [6] and was intensively studied since then (see e.g. [1, 4, 9, 14, 18]). Rather than the usual definition of Frobenius manifolds [2], we prefer the alternative one [4] where Frobenius manifolds are viewed as an enrichment of $F$-manifolds.

Definition 1. i) An F-manifold is a manifold $M$ together with a (fiber preserving) commutative, associative multiplication $\circ$ on $T M$, with unit field 
$e$, and an additional field $E$ (called the Euler field), such that the following conditions hold:

$$
L_{X \circ Y}(\circ)=X \circ L_{Y}(\circ)+Y \circ L_{X}(\circ)
$$

and

$$
L_{E}(\circ)(X, Y)=X \circ Y
$$

for any vector fields $X, Y \in \mathcal{T}_{M}$.

ii) A Frobenius manifold is an F-manifold $(M, \circ, e, E)$ together with a (non-degenerate) flat, multiplication invariant metric $g$ (i.e. $g(X \circ Y, Z)=$ $g(X, Y \circ Z)$, for any $X, Y, Z \in T M)$, such that $L_{E}(g)=D g$ (with $D \in \mathbb{C}$ ) and $\nabla^{\mathrm{LC}}(e)=0$ (where $\nabla^{\mathrm{LC}}$ is the Levi-Civita connection of $g$ ).

iii) A Frobenius metric on an F-manifold $(M, \circ, e, E)$ is a metric $g$ which makes $(M, \circ, e, E, g)$ a Frobenius manifold.

There are examples of $F$-manifolds which do not support locally any Frobenius metric (see Remark 35). In fact, it is difficult to construct explicitly Frobenius manifolds (one strong obstruction being the flatness of the metric). The semisimple case is understood. More precisely, a semisimple $F$-manifold admits, by definition, a coordinate system $\left(u^{i}\right)$, called canonical, in which the multiplication takes the simple form $\partial_{i} \circ \partial_{j}=\delta_{i j} \partial_{j}$. Any multiplication invariant metric is diagonal in canonical coordinates, its flatness is expressed by the Darboux-Egoroff equations and Frobenius metrics exist locally on the open subset $M^{\text {tame }}:=\left\{\left(u^{i}\right), u^{i} \neq u^{j}, i \neq j\right\}$ of tamed points (see e.g. [2]). More general classes of Frobenius manifolds can be obtained from the so called initial condition theorems, developed by Hertling and Manin in [7]. It turns out that the germ $((M, p), \circ, e, E, g)$ of certain Frobenius manifolds is determined (modulo isomorphism) by the linear data induced on the tangent space $T_{p} M$ and conversely, starting with an abstract linear data (called 'initial condition') one obtains a unique (up to isomorphism) germ of such Frobenius manifolds. In the semisimple case, this was already proved in [2], Lecture 3. Generalizations, where the point was replaced by an entire submanifold, were also developed in [7].

We shall be particularly interested in the relation between $F$-manifolds and meromorphic connections. The parameter space $M$ of a meromorphic connection $\nabla$ on a vector bundle over $M \times D$ (where $D \subset \mathbb{C}$ is a small disc around the origin), with poles of Poincaré rank one along $M \times\{0\}$, in Birkhoff normal form $\left(\frac{B_{0}(x)}{\tau}+B_{\infty}\right) \frac{d \tau}{\tau}+\frac{\mathcal{C}_{i}(x) d x^{i}}{\tau}$, inherits (under additional conditions), an $F$-manifold structure. On the other hand, if at a point $x_{0} \in M$ the matrix $B_{0}\left(x_{0}\right)$ is regular (see the comments after Definition 2), then, for a small neighborhood $U$ of $x_{0},\left.\nabla\right|_{U \times D}$ is uniquely determined (up to pullbacks $(f \times \mathrm{Id})^{*}$ and isomorphisms) by its restriction $\nabla^{0}:=\left.\nabla\right|_{\left\{x_{0}\right\} \times D}$. (For 
this reason, $\nabla^{0}$ can be considered as the 'initial condition' for $\nabla$ ). This follows from the existence of a universal integrable deformation $\nabla^{\text {can }}$ of a meromorphic connection (in our case $\nabla^{0}$ ), on a vector bundle over $D$, in Birkhoff normal form, with a pole of Poincaré rank one in $\{0\}$, with regular residue. Such a universal deformation was constructed by Magrange in [10, 11. The parameter space of more general meromorphic connections (not necessarily in the Birkhoff normal form), the so called (TE)-structures, is also an $F$-manifold (see [5]).

In this paper we are concerned with a large class of $F$-manifolds, namely the regular ones, and their relation with Frobenius metrics and meromorphic connections.

Definition 2. An F-manifold $(M, \circ, e, E)$ is called regular if the endomorphism $\mathcal{U}: T M \rightarrow T M, \mathcal{U}(X):=E \circ X$, is regular at any $p \in M$.

(An endomorphism $A: V \rightarrow V$ of a complex vector space $V$ is regular if one of the following equivalent conditions holds: 1) any two distinct Jordan blocks from its Jordan normal form have distinct eigenvalues; 2) the characteristic and minimal polynomials of $A$ coincide; 3$)$ the vector space of endomorphisms of $V$ commuting with $A$ has dimension $n=\operatorname{dim}(V)$ and basis $\left.\left\{\operatorname{Id}, A, \cdots, A^{n-1}\right\} ; 4\right)$ there is a cyclic vector for $A$, i.e. a vector $v \in V$ such that $\left\{v, A(v), \cdots, A^{n-1}(v)\right\}$ is a basis of $\left.V\right)$.

Our main result from this paper is as follows. Its second part can be understood as an initial condition theorem for regular $F$-manifolds.

Theorem 3. i) Any germ $((M, p), \circ, e, E)$ of regular $F$-manifolds is isomorphic to a product $\mathcal{P}:=\Pi_{\alpha=1}^{n}\left(\left(\mathbb{C}^{m_{\alpha}}, 0\right), \circ_{\alpha}, e_{\alpha}, E_{\alpha}\right)$ of germs of (regular) $F$ manifolds. Here $m_{\alpha}$ are the dimensions of the Jordan blocks of the endomorphism $\mathcal{U}_{p}(X)=X \circ E_{p}$ of $T_{p} M$. For each such block, let $a_{\alpha}$ be the corresponding eigenvalue of $\mathcal{U}_{p}$. In the canonical frame field $\left\{\partial_{i}:=\frac{\partial}{\partial t^{i}}, 0 \leq i \leq m_{\alpha}-1\right\}$, determined by coordinates $\left(t^{0}, \cdots, t^{m_{\alpha}-1}\right) \in \mathbb{C}^{m_{\alpha}}$, the multiplication $\circ_{\alpha}$ is given by

$$
\partial_{i} \circ_{\alpha} \partial_{j}=\left\{\begin{array}{l}
\partial_{i+j}, \quad i+j \leq m_{\alpha}-1 \\
0, \quad i+j \geq m_{\alpha},
\end{array}\right.
$$

and the unit field and Euler field by

$$
e_{\alpha}=\partial_{0}, \quad E_{\alpha}=\left(t^{0}+a_{\alpha}\right) \partial_{0}+\left(t^{1}+1\right) \partial_{1}+t^{2} \partial_{2}+\cdots+t^{m_{\alpha}-1} \partial_{m_{\alpha}-1} .
$$

The product $\mathcal{P}$ is canonically associated to $((M, p), \circ, e, E)$ (up to ordering of its factors) and the isomorphism between $((M, p), \circ, e, E)$ and $\mathcal{P}$ is unique (when such an ordering is fixed). 
ii) In particular, there is a unique (up to unique isomorphism) germ of regular $F$-manifolds $((M, p), \circ, e, E)$, with given conjugacy class for the endomorphism $\mathcal{U}_{p}(X):=X \circ E_{p}$ of $T_{p} M$.

(The conjugacy class of an endomorphism is determined by its Jordan normal form; two endomorphisms, defined on not necessarily the same vector space, belong to the same conjugacy class if they can be reduced to the same Jordan normal form).

Structure of the paper. The paper is structured as follows. In Section 2 we recall, following [2, 3, 4, 10, 11, 15], the basic definitions and results we need on Frobenius and $F$-manifolds, Saito bundles and meromorphic connections.

Section 3 represents a first step in the proof of Theorem 3 . Here we prove that a regular $F$-manifold $(M, \circ, e, E)$ for which the endomorphism $\mathcal{U}_{p_{0}}=\mathcal{C}_{E_{p_{0}}} \in \operatorname{End}\left(T_{p_{0}} M\right)$ has exactly one eigenvalue (for $p_{0} \in M$ fixed), is globally nilpotent around $p_{0}$ (see Definition 5 and Proposition 15).

In Section 4 we prove Theorem 3. One can check 'by hand' that each factor $\left(\mathbb{C}^{m_{\alpha}}, \circ_{\alpha}, e_{\alpha}, E_{\alpha}\right)$ in Theorem 3 i) is an $F$-manifold, for which the multiplication at $0 \in \mathbb{C}^{m_{\alpha}}$ by the Euler field $E_{\alpha}$ is a Jordan block in the canonical frame $\left\{\partial_{i}\right\}$ of $\mathbb{C}^{m_{\alpha}}$, with eigenvalue $a_{\alpha}$. This shows the existence of the germ in Theorem 3 ii). In Propositions 18 and 19 from this section we prove the uniqueness of the germ in Theorem 3 ii). The argument is based on Hertling's decomposition of $F$-manifolds [4], the local classification of $\{e\}$-structures [17] and the material from Section 3. The uniqueness of the germ in Theorem 3 ii) implies that any germ $((M, p), \circ, e, E)$ of regular $F$-manifolds is isomorphic to a product $\mathcal{P}$, as required in Theorem $3 \mathrm{i}$ ). The uniqueness of the isomorphisms in Theorem 3 i) and ii) is a consequence of the fact that any automorphism of a germ of regular $F$-manifolds is the identity map (see Lemma 201).

The next sections are devoted to applications of Theorem 3. The local coordinate system on any regular $F$-manifold $(M, \circ, e, E)$, provided by Theorem 3 i), is similar to the canonical coordinate system on semisimple $F$ manifolds. In Section 5 we study Frobenius metrics on $(M, \circ, e, E)$, in these coordinates. We find conditions for the coidentity $e^{b}$ to be closed, the unit $e$ to be flat and, respectively, the Euler field $E$ to preserve the metric (see Proposition 25). The picture is similar to the semisimple case. To express the flatness, we use Dubrovin's description of Frobenius manifolds (without Euler fields) with a maximal abelian group of algebraic symmetries [2]. We find an alternative formulation for this description (see Proposition 23) and we apply it in order to obtain the conditions for a multiplication invariant 
metric on a regular globally nilpotent $F$-manifold to be Frobenius (see Theorem 251). The conditions are more involved than in the semisimple case, owing to the generalized Darboux-Egoroff equations (see Example 26).

In Section 6 we define the Lie algebra of infinitesimal symmetries of a regular $F$-manifold and we compute it using the coordinate system provided by Theorem 3 i) (see Definition and Proposition 291).

In Section 7 we study the relation between regular $F$-manifolds and meromorphic connections. As stated above, the parameter space of certain meromorphic connections are $F$-manifolds, but the converse is not true (not every $F$-manifold can be locally obtained in this way, see Remark 35 b)). We prove that the converse is, however, true, under the regularity assumption (see Corollary [32). Namely, we determine the $F$-manifold structure of the parameter spaces $M^{\text {can }}$ of the Malgrange universal deformations $\nabla^{\text {can }}$, mentioned above, and we show that any regular $F$-manifold is locally isomorphic to such a parameter space (see Proposition 31).

In Section 8 we prove an initial condition theorem for Frobenius metrics on regular $F$-manifolds (see Theorem 34 ). This follows from our Theorem 3. combined with Theorem 4.5 of [7]. While the arguments from [7] work in high generality, they are also quite technical. For completeness of our exposition, we provide in the appendix (Section 9) an alternative, simple and self-contained proof for the existence of the extension of the metric in Theorem 34, based on our treatment of regular $F$-manifolds.

Acknowledgements. L.D. is supported by a Humboldt Research Fellowship. She thanks University of Mannheim for hospitality and the Alexander von Humboldt-Stiftung for financial support. Partial financial support from a CNCS-grant PN-II-ID-PCE-2011-3-0362 is also acknowledged. C.H. acknowledges support by the DFG-grant He 2287/4-1.

\section{Preliminary material}

This section is intended to fix notation. We work in the holomorphic category: the manifolds are complex and the vector bundles, sections, connections etc are holomorphic. We denote by $\mathcal{T}_{M}$ the sheaf of holomorphic vector fields on a complex manifold $M$, by $\mathcal{O}_{M}$ the sheaf of holomorphic functions on $M$ and by $\Omega^{1}(M, V)$ the sheaf of holomorphic 1-forms with values in a vector bundle $V$. In our conventions, the connection form of a connection $\nabla$ on a vector bundle $V \rightarrow M$, in a local basis of sections $\left\{s_{1}, \cdots, s_{n}\right\}$ of $V$, is the matrix valued 1 -form $\Omega=\left(\Omega_{i j}\right)$, defined by $\nabla_{X}\left(s_{i}\right)=\sum_{j=1}^{n}\left(\Omega_{j i}\right)_{X} s_{j}$. The representation of an endomorphism $A \in \operatorname{End}(V)$ (where $V$ is a vec- 
tor space), in a basis $\left\{v_{1}, \cdots, v_{n}\right\}$ of $V$, is the matrix $A=\left(A_{i j}\right)$, where $A\left(v_{i}\right)=\sum_{j=1}^{n} A_{j i} v_{j}$.

\section{$2.1 \quad F$-manifolds}

\subsubsection{Hertling's decomposition of F-manifolds}

The following theorem due to Hertling (see [4, page 16) plays an essential role in the proof of our main result. We shall use it for regular $F$-manifolds, but we remark that the regularity condition is not required for its statement in full generality.

Theorem 4. Let $((M, p), \circ, e, E)$ be a germ of $F$-manifolds and $a_{1}, \cdots, a_{n}$ the distinct eigenvalues of the endomorphism $\mathcal{U}(X):=X \circ E$ of TM at $p$. Then $((M, p), \circ, e, E)$ decomposes into a product $\Pi_{\alpha=1}^{n}\left(\left(M_{\alpha}, p_{\alpha}\right), \circ_{\alpha}, e_{\alpha}, E_{\alpha}\right)$ of germs of $F$-manifolds. For any $1 \leq \alpha \leq n$, the endomorphism $\mathcal{U}_{\alpha}(X):=$ $X \circ_{\alpha} E_{\alpha}$ of $T M_{\alpha}$ has precisely one eigenvalue at $p_{\alpha}$, namely $a_{\alpha}$.

\subsubsection{Basic facts on globally nilpotent $F$-manifolds}

Definition 5. An F-manifold $(M, \circ, e, E)$ is called globally nilpotent if, for any $p \in M$ and $X_{p} \in T_{p} M$, the endomorphism $\mathcal{C}_{X_{p}}: T_{p} M \rightarrow T_{p} M, \mathcal{C}_{X_{p}}\left(Y_{p}\right):=$ $X_{p} \circ Y_{p}$, has exactly one eigenvalue. Equivalently, if

$$
\mathcal{C}_{X_{p}}=\mu\left(X_{p}\right) \mathrm{Id}+N_{X_{p}}
$$

with $\mu\left(X_{p}\right) \in \mathbb{C}$ and $N_{X_{p}} \in \operatorname{End}\left(T_{p} M\right)$ nilpotent.

For any globally nilpotent $F$-manifold $(M, \circ, e, E)$ and $X \in \mathcal{T}_{M}$, the function $\mu(X): M \rightarrow \mathbb{C}, \mu(X)(p):=\mu\left(X_{p}\right)$ is holomorphic. Indeed, the characteristic polynomial $P$ of $\mathcal{C}_{X}$ is given by $P(z, p)=\left(z-\mu\left(X_{p}\right)\right)^{n}$, for any $z \in \mathbb{C}$ and $p \in M$ (where $n=\operatorname{dim}(M))$. Therefore, $\mu(X)=-\frac{1}{n !} P^{(n-1)}(0, \cdot) \in \mathcal{O}_{M}$, as $P$ is holomorphic (the superscript $(n-1)$ denotes the $(n-1)$ derivatives with respect to $z)$. In particular, the eigenfunction $a:=\mu(E)$ of $\mathcal{U}=\mathcal{C}_{E}$ is holomorphic (not necessarily constant).

Recall Definition 2 of regular $F$-manifolds, from the introduction.

Lemma 6. Let $(M, \circ, e, E)$ be a regular $F$-manifold of dimension $n$.

i) For any $k \geq 0$, let $X_{k}:=E \circ \cdots \circ E$ (k-times), with $X_{0}:=e$. The vector fields $\left\{X_{0}, X_{1}, \cdots, X_{n-1}\right\}$ are linear independent (at any point) and

$$
\left[X_{i}, X_{j}\right]=(j-i) X_{i+j-1}, \quad i, j \geq 0 .
$$

ii) Suppose that the endomorphism $\mathcal{U}_{p}\left(X_{p}\right)=X_{p} \circ E_{p}$ of $T_{p} M$ has exactly one eigenvalue, for any $p \in M$. Then $(M, \circ, e, E)$ is globally nilpotent. 
Proof. The linear independence of $\left\{X_{0}, \cdots, X_{n-1}\right\}$ follows from regularity. Formula (44) was proved in [6] (and holds on any $F$-manifold, not necessarily regular). Claim i) follows. We now prove claim ii). By hypothesis, $\mathcal{U}=$ $a$ Id $+N$ on $T M$, where $a \in \mathcal{O}_{M}$ and $N: T M \rightarrow T M$ is nilpotent (at any point). Let $X:=f_{0} X_{0}+\cdots+f_{n-1} X_{n-1} \in \mathcal{T}_{M}$, where $f_{i} \in \mathcal{O}_{M}$. We obtain

$$
\mathcal{C}_{X}=\left(\sum_{k=0}^{n-1} f_{k} a^{k}\right) \mathrm{Id}+\sum_{k=1}^{n-1} f_{k} \sum_{p=1}^{k} C_{k}^{p} a^{k-p} N^{p} .
$$

The second term in the right hand side of the above relation is a nilpotent endomorphism. Our claim follows.

Definition 7. The vector fields $\left\{X_{0}, \cdots, X_{n-1}\right\}$ from Lemma 6 i) form the canonical frame of the regular $F$-manifold $(M, \circ, e, E)$.

\subsubsection{Frobenius metrics on constant $F$-manifolds}

The $F$-manifolds and Frobenius manifolds we are interested in come, by definition, with an Euler field. However, often in the literature the quasihomogeneity condition imposed by the Euler field is considered as an additional obstruction and is not required in the definition of these structures. This is true for example in Dubrovin's description of Frobenius manifolds which underly constant $F$-manifolds [2]. Since we need this description in Subsection 5.2, we recall it here. We begin with the definition of constant $F$-manifolds.

Definition 8. An F-manifold $(N, \circ, e)$ (without Euler field) is called constant if it admits a coordinate system (called canonical) in which the multiplication is constant.

Such an $F$-manifold has an $n$-dimensional abelian group of algebraic symmetries, where $n=\operatorname{dim}(N)$ (see [2], page 69). In a canonical coordinate system $\left(t^{0}, \cdots, t^{n-1}\right), \partial_{i} \circ \partial_{j}=c_{i j}^{k} \partial_{k}$, where $\partial_{i}:=\frac{\partial}{\partial t^{i}}$ and $c_{i j}^{k} \in \mathbb{C}$. (Our notation is different from that used in [2]: in this reference, $\left(t^{i}\right)$ denote the flat coordinates, rather than the canonical ones). We assume that there is a constant (in canonical coordinates), multiplication invariant, (non-degenerate) metric on $T N$ and we fix such a metric $\epsilon$. Using $\epsilon$, we identify $T N$ with $T^{*} N$. The multiplication $\circ$ on $T N$ induces a multiplication, also denoted by $\circ$, on $T^{*} N$. It is given by: $d t^{i} \circ d t^{j}=c_{k}^{i j} d t^{k}$, where $c_{k}^{i j}=\epsilon^{i s} c_{s k}^{j}$. A 1-form $\psi \in \Omega^{1}(N)$ is called invertible if, for any $p \in N$, the covector $\psi_{p} \in T_{p}^{*} N$ is invertible with respect to $\circ$. 
Let $\gamma \in \operatorname{End}(T N)$ be an $\epsilon$ symmetric endomorphism which satisfies the generalized Darboux-Egoroff equations:

$$
\left[\mathcal{C}_{i}, L_{\partial_{j}}(\gamma)\right]-\left[\mathcal{C}_{j}, L_{\partial_{i}}(\gamma)\right]+\left[\left[\mathcal{C}_{i}, \gamma\right],\left[\mathcal{C}_{j}, \gamma\right]\right]=0,0 \leq i, j \leq n-1
$$

where $\mathcal{C}_{i}:=\mathcal{C}_{\partial_{i}}$. Such an endomorphism is called, by analogy with the semisimple case, a rotation coefficient operator. The following description of Frobenius metrics on constant $F$-manifolds is due to Dubrovin (see Theorem 3.1 of [2]). Below $\psi\left[\mathcal{C}_{i}, \gamma\right] \in \Omega^{1}(N)$ is the composition (as maps) of $\left[\mathcal{C}_{i}, \gamma\right] \in$ $\operatorname{End}(T N)$ with the 1 -form $\psi: T N \rightarrow \mathbb{C})$.

Theorem 9. [2] Let $\gamma$ be a rotation coefficient operator on $(N, \circ, e)$ and $\psi \in \Omega^{1}(N)$ invertible, satisfying

$$
L_{\partial_{i}}(\psi)=\psi\left[\mathcal{C}_{i}, \gamma\right], \quad 0 \leq i \leq n-1 .
$$

Then the metric

$$
g(X, Y):=(\psi \circ \psi)(X \circ Y), X, Y \in T N
$$

is Frobenius on $(N, \circ, e)$. Conversely, any Frobenius metric on $(N, \circ, e)$ is of this form, for a certain rotation coefficient operator $\gamma$ and 1 -form $\psi$.

\section{$2.2 \quad F$-manifolds, Frobenius manifolds and Saito bun- dles}

In this paragraph we recall the relation between Frobenius or $F$-manifolds and Saito bundles (see e.g. [15], Chapter VII).

Definition 10. i) A Saito bundle is a vector bundle $\left(\pi: V \rightarrow M, \nabla, \Phi, R_{0}, R_{\infty}\right)$ with a connection $\nabla$, a 1 -form $\Phi \in \Omega^{1}(M, \operatorname{End}(V))$ and two endomorphisms $R_{0}, R_{\infty} \in \operatorname{End}(V)$, such that the following conditions are satisfied:

$$
R^{\nabla}=0, \quad \Phi \wedge \Phi=0, \quad\left[R_{0}, \Phi\right]=0
$$

and

$$
d^{\nabla} \Phi=0, \quad \nabla R_{0}+\Phi=\left[\Phi, R_{\infty}\right], \quad \nabla R_{\infty}=0 .
$$

Above $R^{\nabla}$ is the curvature of $\nabla$ and the $\operatorname{End}(V)$-valued forms $[R, \Phi]$ (with $R:=R_{0}$ or $\left.R_{\infty}\right), d^{\nabla} \Phi$ and $\Phi \wedge \Phi$ are defined by: for any $X, Y \in \mathcal{T}_{M}$,

$$
\begin{aligned}
& {[R, \Phi]_{X}:=\left[R, \Phi_{X}\right]} \\
& \left(d^{\nabla} \Phi\right)_{X, Y}:=\nabla_{X}\left(\Phi_{Y}\right)-\nabla_{Y}\left(\Phi_{X}\right)-\Phi_{[X, Y]} \\
& (\Phi \wedge \Phi)_{X, Y}:=\Phi_{X} \Phi_{Y}-\Phi_{Y} \Phi_{X} .
\end{aligned}
$$


ii) A Saito bundle with metric is a Saito bundle $\left(V, \nabla, \Phi, R_{0}, R_{\infty}\right)$ with a (non-degenerate) metric $g \in S^{2}\left(V^{*}\right)$, such that the following conditions are satisfied:

$$
\nabla g=0, \quad R_{\infty}+R_{\infty}^{*}=0, \quad R_{0}=R_{0}^{*}, \quad \Phi_{X}=\Phi_{X}^{*}, \forall X \in T M,
$$

where the superscript "*"denotes the g-adjoint.

Let $\left(\pi: V \rightarrow M, \nabla, \Phi, R_{0}, R_{\infty}\right)$ be a Saito bundle. Suppose there is a section $s$ of $V$, such that

$$
I: T M \rightarrow V, \quad I(X):=\Phi_{X}(s), X \in T M
$$

is a bundle isomorphism. Define an (associative, commutative, with unit field $\left.e_{M}:=I^{-1}(s)\right)$ multiplication $\circ_{M}$ on $T M$ and a vector field $E_{M} \in \mathcal{T}_{M}$ by the conditions $\Phi_{X{ }_{M} Y}(s)=\Phi_{X} \Phi_{Y}(s)$ and $E_{M}=-I^{-1} R_{0}(s)$. (We remark that our conventions differ from those used in [3, 15]; in these references, the identification between $T M$ and $V$ is done via $-I$; the induced multiplication on $T M$ is then $-\circ_{M}$, but the induced fields $E_{M}$ are the same). The following holds (see e.g. Lemmas 4.1 and 4.3 of [3]):

Proposition 11. Both $\circ_{M}$ and $E_{M}$ are independent of the section $s$ and $\left(M, \circ_{M}, e_{M}, E_{M}\right)$ is an F-manifold. The endomorphism $\mathcal{U}_{M}(X)=X \circ E_{M}$ of $T M$ coincides with $-I^{-1} R_{0} I$. In particular, $\left(\mathcal{U}_{M}\right)_{p}$ and $-\left(R_{0}\right)_{p}$ belong to the same conjugacy class, for any $p \in M$.

Suppose now that $g$ is a metric on $V$, which makes $\left(V, \nabla, \Phi, R_{0}, R_{\infty}, g\right)$ a Saito bundle with metric. Suppose that the section $s$ from the isomorphism (9) is $\nabla$-parallel and $R_{\infty}(s)=q s$, for $q \in \mathbb{C}$. Such a section is called primitive homogeneous. Then $g_{M}(X, Y):=g(I(X), I(Y))$ is a Frobenius metric on $\left(M, \circ_{M}, e_{M}, E_{M}\right)$. The Levi-Civita $\nabla^{\mathrm{LC}}$ of $g_{M}$ is given by $\nabla^{\mathrm{LC}}=I^{-1} \circ \nabla \circ I$ and

$$
\nabla^{\mathrm{LC}} E_{M}=I^{-1} R_{\infty} I+(1-q) \mathrm{Id} .
$$

(see [15], page 239). Conversely, any Frobenius manifold arises in this way (see [15], page 240).

\subsection{F-manifolds and flat meromorphic connections}

Let $\nabla$ be a flat meromorphic connection on a vector bundle $E$ over $M \times D$ (where $D \subset \mathbb{C}$ is a small disc around the origin), with poles of Poincaré rank one along $M \times\{0\}$, in Birkhoff normal form. By definition, this means that 
$E=(M \times D) \times \mathbb{C}^{n} \rightarrow M \times D$ is the trivial bundle and the connection form of $\nabla$ in the standard trivialization of $E$ is given by

$$
\Omega=\left(\frac{B_{0}(x)}{\tau}+B_{\infty}\right) \frac{d \tau}{\tau}+\frac{\mathcal{C}_{i}(x) d x^{i}}{\tau}
$$

where $\left(x^{i}\right)$ are coordinates on $M, \tau$ is the coordinate on $D, \mathcal{C}_{i}: M \rightarrow M_{n}(\mathbb{C})$, $B_{0}: M \rightarrow M_{n}(\mathbb{C})$ and $B_{\infty} \in M_{n}(\mathbb{C})$. We consider $B_{0}, B_{\infty}$ as endomorphisms (the latter, constant) of the trivial bundle $V=M \times \mathbb{C}^{n} \rightarrow M$ and $\mathcal{C}=\mathcal{C}_{i} d x^{i}$ as an $\operatorname{End}(V)$-valued 1-form on $M$. In its simplest form, the relation between $F$-manifolds and meromorphic connections is the following (for the more general relation between $F$-manifolds and (TE)-structures, see [5], Theorem $3.1)$.

Proposition 12. Let $\nabla$ be a flat meromorphic connection on the trivial bundle $E=(M \times D) \times \mathbb{C}^{n} \rightarrow M \times D$, in Birkhoff normal form (11).

i) The trivial bundle $V=M \times \mathbb{C}^{n} \rightarrow M$, together with $\left(D, \mathcal{C}, B_{0},-B_{\infty}\right)$, where $D$ is the canonical flat connection of $V$ and $\mathcal{C}, B_{0}, B_{\infty}$ are as above, is a Saito bundle.

ii) In particular, if there is a section $s$ of $V$, such that (9) is an isomorphism, then $M$ inherits an F-manifold structure.

Proof. Claim i) follows from the flatness condition $d \Omega+\Omega \wedge \Omega=0$. Claim ii) follows from Proposition 11 .

\subsection{Malgrange universal deformation}

Let $\nabla^{0}$ be a connection on the trivial bundle $V^{0}=D \times \mathbb{C}^{n} \rightarrow D$, with connection form

$$
\Omega^{0}=\left(\frac{B_{0}^{o}}{\tau}+B_{\infty}\right) \frac{d \tau}{\tau},
$$

where $B_{0}^{o}, B_{\infty} \in M_{n}(\mathbb{C})$. To keep the text self-contained, we recall the definition of an integrable deformation of $\nabla^{0}$.

Definition 13. An integrable deformation of $\nabla^{0}$ is a flat meromorphic connection $\nabla$ on the trivial vector bundle $E=(M \times D) \times \mathbb{C}^{n} \rightarrow M \times D$, in Birkhoff normal form (11), which coincides with $\nabla^{0}$ when restricted to $\left\{p_{0}\right\} \times D\left(\right.$ where $\left.p_{0} \in M\right)$.

Assume now that $B_{0}^{o}$ is regular. Then $\nabla^{0}$ admits an integrable deformation $\nabla^{\text {can }}$, constructed by Malgrange [10, 11], which is universal (see e.g. [15], page 208 , for the definition of universal integrable deformations). Following 
Sabbah ([15], Chapter VI, Section 3.a), we now recall its construction. Let $\mathcal{D} \subset T\left(M_{n}\left(\mathbb{C}^{n}\right)\right)$ be defined by

$$
\mathcal{D}_{\Gamma}:=\operatorname{Span}_{\mathbb{C}}\left\{\operatorname{Id},\left(B_{0}\right)_{\Gamma}, \cdots,\left(B_{0}\right)_{\Gamma}^{n-1}\right\} \subset T_{\Gamma} M_{n}(\mathbb{C})=M_{n}(\mathbb{C}),
$$

where

$$
\left(B_{0}\right)_{\Gamma}:=B_{0}^{o}-\Gamma+\left[B_{\infty}, \Gamma\right] .
$$

Because $B_{0}^{o}$ is regular, so is $\left(B_{0}\right)_{\Gamma}$, for any $\Gamma \in W$, where $W$ is a small open neighborhood of 0 in $M_{n}(\mathbb{C})$. For any $\Gamma \in W, \mathcal{D}_{\Gamma}$ is the (n-dimensional) vector space of polynomials in $\left(B_{0}\right)_{\Gamma}$ and the distribution $\mathcal{D} \rightarrow W$ is integrable. The Malgrange universal deformation of $\nabla^{0}$ is defined as follows [10, 11] (also [15]):

Definition 14. i) The parameter space $M^{\text {can }}=M^{\text {can }}\left(B_{0}^{o}, B_{\infty}\right)$ of the universal deformation $\nabla^{\text {can }}$ of $\nabla^{0}$ is the maximal integral submanifold of $\left.\mathcal{D}\right|_{W}$, passing through 0.

ii) The connection $\nabla^{\text {can }}$ of $\nabla^{0}$ is defined on the trivial bundle $E=\left(M^{\mathrm{can}} \times\right.$ D) $\times \mathbb{C}^{n} \rightarrow M^{\text {can }} \times D$, with connection form in the standard trivialization of E given by

$$
\Omega^{\mathrm{can}}=\left(\frac{B_{0}}{\tau}+B_{\infty}\right) \frac{d \tau}{\tau}+\frac{\mathcal{C}}{\tau} .
$$

Here $B_{0}: M^{\text {can }} \rightarrow M_{n}(\mathbb{C}),\left(B_{0}\right)(\Gamma):=\left(B_{0}\right)_{\Gamma}$ is given by (14) and $\mathcal{C}_{X}:=X$ is the action of the matrix $X$ on $\mathbb{C}^{n}$, for any $X \in T_{\Gamma} M^{\text {can }} \subset M_{n}(\mathbb{C})$.

\section{Globally nilpotent regular F-manifolds}

Our aim in this section is to prove the following result.

Proposition 15. Let $(M, \circ, e, E)$ be an F-manifold of dimension $n \geq 2$, such that at a point $p_{0} \in M$, the endomorphism $\mathcal{U}_{p_{0}}=\left(\mathcal{C}_{E}\right)_{p_{0}}: T_{p_{0}} M \rightarrow T_{p_{0}} M$ is regular, with exactly one eigenvalue. Then there is a neighborhood $U$ of $p_{0}$, such that $(U, \circ, e, E)$ is globally nilpotent (and regular).

Proof. Let $U$ be a small neighborhood of $p_{0}$, such that, for any $p \in U$, the endomorphism $\mathcal{U}_{p}=\left(\mathcal{C}_{E}\right)_{p}: T_{p} M \rightarrow T_{p} M$ is regular, and let $P(p, z)=$ $z^{n}+\sum_{k=0}^{n-1} \lambda_{k}(p) z^{k}$ be the characteristic (or minimal) polynomial of $\mathcal{U}_{p}$. Denote by $\left\{X_{0}, X_{1}, \cdots, X_{n-1}\right\}$ the canonical frame of the regular $F$-manifold $(U, \circ, e, E)$ (see Definition 7). Since $P\left(p, \mathcal{U}_{p}\right)=0$,

$$
X_{n}+\sum_{k=0}^{n-1} \lambda_{k} X_{k}=0
$$


Define the functions

$$
f_{k}:=\lambda_{k}-\frac{C_{n}^{k}}{n^{n-k}} \lambda_{n-1}^{n-k}, \quad 0 \leq k \leq n-2 .
$$

(Remark that $f_{k}(p)=0$ for $p \in U$ and all $0 \leq k \leq n-2$, if and only if $P(p, z)=\left(z+\frac{\lambda_{n-1}(p)}{n}\right)^{n}$, if and only if $\mathcal{U}_{p}$ has exactly one eigenvalue). By hypothesis, $f_{k}\left(p_{0}\right)=0$, for any $0 \leq k \leq n-2$. Our aim is to compute the derivatives $X_{i}\left(f_{k}\right)$ and to show, using the Cauchy-Kovalevskaia theorem, that $f_{k}=0$ on $U$, for any $0 \leq k \leq n-2$. This implies that $\mathcal{U}$ has exactly one eigenvalue at any point of $U$ and we conclude from Lemma 6 that $(U, \circ, e, E)$ is globally nilpotent, as required. Details are as follows.

We take the Lie derivative of (16) with respect to $X_{s}(s \geq 0)$ and we use (41). We obtain

$$
(n-s) X_{s+n-1}+\sum_{k=0}^{n-1}\left(X_{s}\left(\lambda_{k}\right) X_{k}+(k-s) \lambda_{k} X_{s+k-1}\right)=0,
$$

which is equivalent, by taking $\lambda_{n}:=1$, to

$$
\sum_{k=0}^{n-1} X_{s}\left(\lambda_{k}\right) X_{k}+\sum_{k=0}^{n}(k-s) \lambda_{k} X_{s+k-1}=0
$$

Relation (18), with $s=0$, gives

$$
X_{0}\left(\lambda_{k}\right)=-(k+1) \lambda_{k+1}, 0 \leq k \leq n-1 .
$$

Relation (18), with $s=1$, gives

$$
\sum_{k=0}^{n-1} X_{1}\left(\lambda_{k}\right) X_{k}+\sum_{k=0}^{n}(k-1) \lambda_{k} X_{k}=0
$$

From (16), $X_{n}=-\sum_{k=0}^{n-1} \lambda_{k} X_{k}$. Replacing this expression of $X_{n}$ into the above relation we obtain

$$
X_{1}\left(\lambda_{k}\right)=(n-k) \lambda_{k}, \quad 0 \leq k \leq n-1 .
$$

The computation of $X_{2}\left(\lambda_{k}\right)$ is done in the same way, but is a bit more complicated. Taking in (18) $s=2$ we obtain

$$
\sum_{k=0}^{n-1} X_{2}\left(\lambda_{k}\right) X_{k}+\sum_{k=0}^{n}(k-2) \lambda_{k} X_{k+1}=0
$$


or, equivalently,

$$
\sum_{k=0}^{n-1} X_{2}\left(\lambda_{k}\right) \mathcal{U}^{k}+\sum_{k=0}^{n}(k-2) \lambda_{k} \mathcal{U}^{k+1}=0 .
$$

Since $P(p, \cdot)$ is the minimal polynomial of $\mathcal{U}_{p}$ (for any $p \in U$ ) there are holomorphic functions $b_{0}, b_{1} \in \mathcal{O}_{U}$ such that

$$
\sum_{k=0}^{n-1} X_{2}\left(\lambda_{k}\right) z^{k}+\sum_{k=0}^{n}(k-2) \lambda_{k} z^{k+1}=\left(b_{0}+b_{1} z\right) \sum_{k=0}^{n} \lambda_{k} z^{k} .
$$

Identifying in (21) the coefficients of $z^{n+1}$ we obtain $b_{1}=n-2$. Relation (21) becomes

$$
\sum_{k=0}^{n}\left(X_{2}\left(\lambda_{k}\right)-b_{0} \lambda_{k}\right) z^{k}-\sum_{k=1}^{n}(n-k+1) \lambda_{k-1} z^{k}=0 .
$$

Then (from the coefficient of $\left.z^{0}\right), X_{2}\left(\lambda_{0}\right)=b_{0} \lambda_{0}$, and the remaining terms in (22) give

$$
\sum_{k=1}^{n}\left(X_{2}\left(\lambda_{k}\right)-b_{0} \lambda_{k}-(n-k+1) \lambda_{k-1}\right) z^{k}=0 .
$$

Using $\lambda_{n}=1$, we obtain $b_{0}=-\lambda_{n-1}$ (from the coefficient of $z^{n}$ ) and relation (23) becomes

$$
X_{2}\left(\lambda_{k}\right)=-\lambda_{n-1} \lambda_{k}+(n-k+1) \lambda_{k-1}, 0 \leq k \leq n-1 .
$$

(We use the convention $\lambda_{i}=0$ for $i<0$; similarly, below $f_{i}=0$ whenever $i<0)$.

Next, we compute the derivatives $X_{3}\left(\lambda_{k}\right)$. Relation (18), with $s=3$, gives, by a similar argument as for $s=2$,

$$
X_{3}\left(\lambda_{k}\right)=\left(\lambda_{n-1}^{2}-2 \lambda_{n-2}\right) \lambda_{k}+(n-k+2) \lambda_{k-2}-\lambda_{n-1} \lambda_{k-1}, 0 \leq k \leq n-1 .
$$

From relations (19), (20), (24) and (25), we obtain, from long but straightforward computations, the expressions for the derivatives $X_{i}\left(f_{k}\right)$ (for any $0 \leq i \leq 3$ and $0 \leq k \leq n-2)$ :

$$
X_{0}\left(f_{k}\right)=-(k+1) f_{k+1}(k \leq n-3), X_{0}\left(f_{n-2}\right)=0, \quad X_{1}\left(f_{k}\right)=(n-k) f_{k}
$$

and

$$
\begin{aligned}
& X_{2}\left(f_{k}\right)=-\lambda_{n-1} f_{k}+(n-k+1) f_{k-1}-\frac{2 C_{n}^{k}(n-k)}{n^{n-k}} \lambda_{n-1}^{n-k-1} f_{n-2} \\
& X_{3}\left(f_{k}\right)=\left(\lambda_{n-1}^{2}-2 \lambda_{n-2}\right) f_{k}+\frac{C_{n}^{k}(3 n-3 k-2)}{n^{n-k}} \lambda_{n-1}^{n-k} f_{n-2} \\
& +(n-k+2) f_{k-2}-\lambda_{n-1} f_{k-1}-\frac{3 C_{n}^{k}(n-k)}{n^{n-k}} \lambda_{n-1}^{n-k-1} f_{n-3} .
\end{aligned}
$$


In particular, both (26) and (27) are of the form

$$
X_{i}\left(f_{k}\right)=\sum_{s=0}^{n-2} a_{k s}^{(i)} f_{s}, 0 \leq i \leq 3,0 \leq k \leq n-2,
$$

for some $a_{k s}^{(i)} \in \mathcal{O}_{U}$. Since $\left[X_{i}, X_{j}\right]=(j-i) X_{i+j-1}$ (see relation (44)), we obtain that the derivatives $X_{i}\left(f_{k}\right)$, for any $0 \leq i \leq n-1$ (and $0 \leq k \leq n-2$ ), are of the form (28). In a coordinate chart $\chi=\left(y^{0}, \cdots, y^{n-1}\right): U \rightarrow \mathbb{C}^{n}$ with $\chi\left(p_{0}\right)=0$ we obtain

$$
\frac{\partial\left(f_{k} \circ \chi^{-1}\right)}{\partial y^{i}}=\sum_{s=0}^{n-2} b_{k s}^{(i)}\left(f_{s} \circ \chi^{-1}\right), \quad 0 \leq i \leq n-1, \quad 0 \leq k \leq n-2,
$$

for some $b_{k s}^{(i)} \in \mathcal{O}_{\chi(U)}$. Also, $\left(f_{k} \circ \chi^{-1}\right)(0)=0$ for any $0 \leq k \leq n-2$. Applying successively the uniqueness statement of the Cauchy-Kovalevskaia theorem (in the form stated e.g. in [7], relations (2.42) and (2.43), with no $\left(t^{i}\right)$-parameters in the notation of this reference), we obtain that $f_{k}=0$ on $U$, as required.

The computations from the above proof imply the following corollary.

Corollary 16. Let $(M, \circ, e, E)$ be an n-dimensional globally nilpotent regular $F$-manifold and $\left\{X_{0}, \cdots, X_{n-1}\right\}$ its canonical frame. Let $a \in \mathcal{O}_{M}$ be the eigenfunction of $\mathcal{U}=\mathcal{C}_{E}$. Then

$$
\left[X_{i}, X_{j}\right]=\left\{\begin{array}{l}
(j-i) X_{i+j-1}, \quad i+j \leq n \\
(i-j) \sum_{k=0}^{n-1} c_{k}^{(i+j-1-n)} a^{i+j-1-k} X_{k}, \quad i+j>n,
\end{array}\right.
$$

where $c_{k}^{(p)}(p \geq 0$ and $0 \leq k \leq n-1)$ are constants, defined inductively by $c_{k}^{(0)}=(-1)^{n-k} C_{n}^{k}$ and for any $s \geq 0, c_{k}^{(s+1)}=c_{k-1}^{(s)}-c_{k}^{(0)} c_{n-1}^{(s)}($ when $k \geq 1)$ and $c_{0}^{(s+1)}=-c_{0}^{(0)} c_{n-1}^{(s)}$. Moreover,

$$
X_{i}(a)=a^{i}, \quad i \geq 0 .
$$

Proof. Relation (29) for $i+j \leq n$ is just (44). We now prove (29) for $i+j>n$. Since $(\mathcal{U}-a \mathrm{Id})^{n}=0$,

$$
\mathcal{U}^{n}+\sum_{k=0}^{n-1} c_{k}^{(0)} a^{n-k} \mathcal{U}^{k}=0
$$


Multiplying the above relation with $\mathcal{U}, \mathcal{U}^{2}$, etc, and using an induction argument, we obtain

$$
\mathcal{U}^{n+s}+\sum_{k=0}^{n-1} c_{k}^{(s)} a^{n-k+s} \mathcal{U}^{k}=0, \quad s \geq 0
$$

or, equivalently,

$$
X_{n+s}=-\sum_{k=0}^{n-1} c_{k}^{(s)} a^{n-k+s} X_{k}, \quad s \geq 0 .
$$

Relations (44) and (32) imply (29) for $i+j>n$, as required.

It remains to prove (30). With the notation from the proof of Proposition 15, $a=-\frac{\lambda_{n-1}}{n}$ and $\lambda_{k}=C_{n}^{k}(-a)^{n-k}$, for any $0 \leq k \leq n-1$ (because $f_{k}=0$, $(M, \circ, e, E)$ being globally nilpotent). On the other hand, in the proof of Proposition 15 we computed the following derivatives:

$$
\begin{aligned}
& X_{0}\left(\lambda_{n-1}\right)=-n, X_{1}\left(\lambda_{n-1}\right)=\lambda_{n-1} \\
& X_{2}\left(\lambda_{n-1}\right)=-\lambda_{n-1}^{2}+2 \lambda_{n-2} \\
& X_{3}\left(\lambda_{n-1}\right)=\lambda_{n-1}^{3}+3 \lambda_{n-3}-3 \lambda_{n-1} \lambda_{n-2} .
\end{aligned}
$$

These expressions, written in terms of $a$, give (30), for $0 \leq i \leq 3$. Using (41) we obtain (30), for any $i \geq 0$.

Remark 17. For the proof of Theorem 3 (next section), it is convenient to express the Lie brackets $\left[X_{i}, X_{j}\right]$, computed in Corollary 16, in a unified form (not as in (29), where the cases $i+j \leq n$ and $i+j>n$ are separated). This can be done as follows. Consider the constants $c_{k}^{(p)}$ from Corollary 16. They were defined for $p \geq 0$ and $0 \leq k \leq n-1$. For $p<0$ (and $0 \leq k \leq n-1$ ), let $c_{k}^{(p)}:=0$, unless $p=k-n$, in which case $c_{k}^{(k-n)}:=-1$. With this notation, the two relations (29) reduce to the single one

$$
\left[X_{i}, X_{j}\right]=(i-j) \sum_{k=0}^{n-1} c_{k}^{(i+j-1-n)} a^{i+j-1-k} X_{k}, \quad i, j \geq 0
$$

\section{Proof of Theorem 3}

Using the material from the previous section, we now prove Theorem 3. With the explanations from the introduction, the only statements which need to be proved are the uniqueness (up to isomorphism) of the germ in Theorem 3 ii) and the uniqueness of the isomorphisms in Theorem 3 i) and ii). 
We begin by proving the uniqueness of the germ. Consider two germs $\left((M, p), \circ_{M}, e_{M}, E_{M}\right)$ and $\left((N, q), \circ_{N}, e_{N}, E_{N}\right)$ of $n$-dimensional $F$-manifolds. Let $\mathcal{U}_{M} \in \operatorname{End}(T M)$ and $\mathcal{U}_{N} \in \operatorname{End}(T N)$ be the endomorphisms given by the multiplication with the Euler fields. We assume that $\left(\mathcal{U}_{M}\right)_{p}: T_{p} M \rightarrow T_{p} M$ and $\left(\mathcal{U}_{N}\right)_{q}: T_{q} N \rightarrow T_{q} N$ are regular and belong to the same conjugacy class. Our aim is to show that the two germs are isomorphic. Owing to Hertling's decomposition of $F$-manifolds (see Theorem 44), we can (and will) assume that $\left(\mathcal{U}_{M}\right)_{p}$ and $\left(\mathcal{U}_{N}\right)_{q}$ have exactly one eigenvalue. From Proposition 15, the germs $\left((M, p), \circ_{M}, e_{M}, E_{M}\right)$ and $\left((N, q), \circ_{N}, e_{N}, E_{N}\right)$ are globally nilpotent. Let $a \in \mathcal{O}_{M}$ and $b \in \mathcal{O}_{N}$ be the eigenfunction of $\mathcal{U}_{M}$ and $\mathcal{U}_{N}$, respectively. Since $\left(\mathcal{U}_{M}\right)_{p}$ and $\left(\mathcal{U}_{N}\right)_{q}$ belong to the same conjugacy class, $a(p)=b(q)$. We denote by $\left\{X_{i}:=\left(E_{M}\right)^{i}, 0 \leq i \leq n-1\right\}$ and $\left\{Y_{i}:=\left(E_{N}\right)^{i}, 0 \leq i \leq n-1\right\}$ the canonical frames of the two germs.

Proposition 18. In the above setting, there is a biholomorphic transformation $\psi:(M, p) \rightarrow(N, q)$, such that $\psi_{*}\left(X_{i}\right)=Y_{i}$ for any $0 \leq i \leq n-1$ and $b \circ \psi=a$.

Proof. The statement follows from the classification of $\{e\}$-structures (see Theorem 4.1 of [17], page 344). For completeness of our exposition we present the argument in detail, by adapting the proof of Theorem 4.1 of [17] to our setting. Let $\left\{\omega_{i}^{X} 0 \leq i \leq n-1\right\}$ and $\left\{\omega_{i}^{Y}, 0 \leq i \leq n-1\right\}$ be the 1-forms dual to $\left\{X_{i}, 0 \leq i \leq n-1\right\}$ and $\left\{Y_{i}, 0 \leq i \leq n-1\right\}$, respectively. From Remark [17,

$$
d \omega_{k}^{X}=\sum_{0 \leq i, j \leq n-1}\left(c^{X}\right)_{i j}^{k} \omega_{i}^{X} \wedge \omega_{j}^{X}, \quad\left(c^{X}\right)_{i j}^{k}:=-\frac{1}{2}(i-j) c_{k}^{(i+j-1-n)} a^{i+j-1-k}
$$

and similarly

$$
d \omega_{k}^{Y}=\sum_{0 \leq i, j \leq n-1}\left(c^{Y}\right)_{i j}^{k} \omega_{i}^{Y} \wedge \omega_{j}^{Y}, \quad\left(c^{Y}\right)_{i j}^{k}:=-\frac{1}{2}(i-j) c_{k}^{(i+j-1-n)} b^{i+j-1-k} .
$$

From relation (30),

$$
d a=\sum_{i=0}^{n-1} a^{i} \omega_{i}^{X}, \quad d b=\sum_{i=0}^{n-1} b^{i} \omega_{i}^{Y}
$$

In particular, $d_{p} a \in T_{p}^{*} M$ and $d_{q} b \in T_{q}^{*} N$ are non-trivial. We restrict $M$ and $N$ such that $d_{x} a \neq 0$ and $d_{y} b \neq 0$, for any $x \in M$ and $y \in N$. Let $\pi_{1}: M \times N \rightarrow M$ and $\pi_{2}: M \times N \rightarrow N$ be the natural projections, 
$\theta_{i}^{X}:=\left(\pi_{1}\right)^{*}\left(\omega_{i}^{X}\right)$ and $\theta_{i}^{Y}:=\left(\pi_{2}\right)^{*}\left(\omega_{i}^{Y}\right)$ for any $i$. Pulling back (35) to $M \times N$, we obtain

$$
d\left(\pi_{1}^{*} a\right)=\sum_{i=0}^{n-1}\left(\pi_{1}^{*} a\right)^{i} \theta_{i}^{X}, \quad d\left(\pi_{2}^{*} b\right)=\sum_{i=0}^{n-1}\left(\pi_{2}^{*} b\right)^{i} \theta_{i}^{Y} .
$$

Let $S$ be the $(2 n-1)$-dimensional submanifold of $M \times N$, defined by

$$
S:=\{(x, y) \in M \times N, a(x)=b(y)\} .
$$

Remark that $(p, q) \in S$. Let $\vec{i}: S \rightarrow M \times N$ be the inclusion. We will show that

$$
\mathcal{D}_{S}:=\operatorname{Span}\left\{(\vec{i})^{*}\left(\theta_{i}^{X}-\theta_{i}^{Y}\right), 0 \leq i \leq n-1\right\} \subset T^{*} S
$$

is a rank $(n-1)$ distribution on $S$. For this, we remark from (36) that

$$
\theta_{0}^{X}-\theta_{0}^{Y}=d\left(\pi_{1}^{*} a-\pi_{2}^{*} b\right)-\sum_{i=1}^{n-1}\left(\left(\pi_{1}^{*} a\right)^{i} \theta_{i}^{X}-\left(\pi_{2}^{*} b\right)^{i} \theta_{i}^{Y}\right)
$$

Restricting (37) to TS and using that $\pi_{1}^{*} a=\pi_{2}^{*} b$ on $S\left(\right.$ and $d\left(\pi_{1}^{*} a-\pi_{2}^{*} b\right)=0$ on $T S$ ), we obtain that $(\vec{i})^{*}\left(\theta_{0}^{X}-\theta_{0}^{Y}\right)$ is a linear combination of the remaining $(\vec{i})^{*}\left(\theta_{i}^{X}-\theta_{i}^{Y}\right), 1 \leq i \leq n-1$. We deduce that $\operatorname{rank}\left(\mathcal{D}_{S}\right) \leq n-1$. On the other hand, since $S$ is of codimension 1 in $M \times N$, the kernel of the map $(\vec{i})^{*}$ : $T_{(x, y)}^{*}(M \times N) \rightarrow T_{(x, y)}^{*} S$ is one dimensional (for any $(x, y) \in S$ ). We deduce that the kernel of the restriction of this map to $\operatorname{Span}\left\{\left(\theta_{i}^{X}-\theta_{i}^{Y}\right)_{(x, y)}, 0 \leq i \leq\right.$ $n-1\} \subset T_{(x, y)}^{*}(M \times N)$ is at most one dimensional. Since $\left\{\left(\theta_{i}^{X}-\theta_{i}^{Y}\right)_{(x, y)}\right\}$ are linearly independent, we obtain that $\operatorname{dim}\left(\mathcal{D}_{S}\right)_{(x, y)} \geq n-1$. We conclude that $\operatorname{dim}\left(\mathcal{D}_{S}\right)_{(x, y)}=n-1$, for any $(x, y) \in S$, i.e. $\mathcal{D}_{S}$ is of rank $n-1$, as needed.

We now prove that the distribution $\mathcal{D}_{S}$ is involutive. Pulling back the first relations (33) and (34) to $S$, and using $\pi_{1}^{*}\left(c^{X}\right)_{i j}^{k}=\pi_{2}^{*}\left(c^{Y}\right)_{i j}^{k}$ on $S$ (which follows from the definition of $\left(c^{X}\right)_{i j}^{k}$ and $\left(c^{Y}\right)_{i j}^{k}$ and from $\pi_{1}^{*} a=\pi_{2}^{*} b$ on $S$ ), we obtain that

$d\left(\theta_{k}^{X}-\theta_{k}^{Y}\right)=\sum_{i, j} \pi_{1}^{*}\left(c^{X}\right)_{i j}^{k}\left(\theta_{i}^{X}-\theta_{i}^{Y}\right) \wedge \theta_{j}^{X}+\sum_{i, j} \pi_{2}^{*}\left(c^{X}\right)_{i j}^{k} \theta_{i}^{Y} \wedge\left(\theta_{j}^{X}-\theta_{j}^{Y}\right), \quad \forall k$

i.e. $\mathcal{D}_{S}$ is an integrable distribution on $S$.

Consider now the integral submanifold $S^{\prime}$ of $\mathcal{D}_{S}$ which contains $(p, q)$. It is of $\operatorname{dimension} \operatorname{dim}(S)-\operatorname{rank}\left(\mathcal{D}_{S}\right)=(2 n-1)-(n-1)=n$. We claim that $\left\{\left.\left(\theta_{i}^{X}\right)\right|_{T S^{\prime}}, 0 \leq i \leq n-1\right\}$ is a basis of forms on $S^{\prime}$. Indeed, since the forms $\left\{\left(\theta_{i}^{X}\right)_{(x, y)},\left(\theta_{i}^{\bar{X}}-\theta_{i}^{\bar{Y}}\right)_{(x, y)}, 0 \leq i \leq n-1\right\}$ are a basis of $T_{(x, y)}^{*}(M \times N)$, their restriction to $T_{(x, y)} S^{\prime}$ generate $T_{(x, y)}^{*} S^{\prime}$. Therefore, $\left\{\left.\left(\theta_{i}^{X}\right)\right|_{T_{(x, y)} S^{\prime}}, 0 \leq i \leq n-1\right\}$ 
generate $T_{(x, y)}^{*} S^{\prime}$, hence form a basis of $T_{(x, y)}^{*} S^{\prime}$ (because $\left(\theta_{i}^{X}-\theta_{i}^{Y}\right)_{(x, y)}$ vanishes on $T_{(x, y)} S^{\prime}=\left(\mathcal{D}_{S}\right)_{(x, y)}$ and $\left.\operatorname{dim}\left(S^{\prime}\right)=n\right)$. We proved that $\left\{\left.\left(\theta_{i}^{X}\right)\right|_{T S^{\prime}}, 0 \leq\right.$ $i \leq n-1\}$ is a basis of forms on $S^{\prime}$, as needed. From this fact and $\left(\left.\pi_{1}\right|_{S^{\prime}}\right)^{*}\left(\omega_{i}^{X}\right)=\left.\left(\theta_{i}^{X}\right)\right|_{T S^{\prime}}$, we obtain that $\left.\pi_{1}\right|_{S^{\prime}}: S^{\prime} \rightarrow M$ is locally a biholomorphic transformation. A similar argument shows that $\left.\pi_{2}\right|_{S^{\prime}}: S^{\prime} \rightarrow N$ is also, locally, a biholomorphic transformation. We restrict the representatives $M$ and $N$ of the germs, such that $\pi_{1}: S^{\prime} \rightarrow M$ and $\pi_{2}: S^{\prime} \rightarrow N$ are biholomorphic transformations and we define $\psi:=\pi_{2} \circ \pi_{1}^{-1}$. Since $(p, q) \in S^{\prime}, \psi(p)=q$. Since any $(x, y) \in S^{\prime}$ satisfies $a(x)=b(y)$, we obtain that $b \circ \psi=a$. Since $\pi_{1}^{*}\left(\omega_{i}^{X}\right)=\pi_{2}^{*}\left(\omega_{i}^{Y}\right)$ on $T S^{\prime}$, we obtain that $\psi^{*}\left(\omega_{i}^{Y}\right)=\omega_{i}^{X}$, i.e. $\psi_{*}\left(X_{i}\right)=Y_{i}$, for any $0 \leq i \leq n-1$.

Proposition 19. The map $\psi:\left((M, p), \circ_{M}, e_{M}, E_{M}\right) \rightarrow\left((N, q), \circ_{N}, e_{N}, E_{N}\right)$ from Proposition 18 is an isomorphism of germs of F-manifolds.

Proof. From Proposition 18 the map $\psi$ preserves the unit and Euler fields. It remains to check that it preserves the multiplications, too. From regularity, this is equivalent to $\psi_{*}\left(E_{M}^{i}\right)=E_{N}^{i}$, for any $i \geq 0$. The statement for $i \leq n-1$ follows from Proposition 18 (since $E_{M}^{i}=X_{i}$ and $E_{N}^{i}=Y_{i}$ for such $i$ ). We need to prove that $\psi_{*}\left(E_{M}^{i}\right)=E_{N}^{i}$ also for $i \geq n$. For this, we notice that the characteristic polynomials of $\left(\mathcal{U}_{M}\right)_{x}$ and $\left(\mathcal{U}_{N}\right)_{\psi(x)}$ coincide, for any $x \in M$ (both $\left(\mathcal{U}_{M}\right)_{x}$ and $\left(\mathcal{U}_{N}\right)_{\psi(x)}$ are regular, defined on vector spaces of the same dimension, with the same (unique) eigenvalue $a(x)=(b \circ \psi)(x))$. Therefore, for any $i \geq n$, the coordinates of $\left(E_{M}\right)_{x}^{i}$ in the basis $\left\{\left(X_{j}\right)_{x}, 0 \leq j \leq n-1\right\}$ coincide with the coordinates of $\left(E_{N}\right)_{\psi(x)}^{i}$ in the basis $\left\{\left(Y_{j}\right)_{\psi(x)}, 0 \leq j \leq\right.$ $n-1\}$. Using that $\psi_{*}\left(X_{j}\right)=Y_{j}$ we deduce that $\psi_{*}\left(\left(E_{M}^{i}\right)_{x}\right)=\left(E_{N}^{i}\right)_{\psi(x)}$, i.e. $\psi_{*}\left(E_{M}^{i}\right)=E_{N}^{i}$, as needed.

The uniqueness of the isomorphisms required by Theorem 3 i) and ii) is a consequence of the following simple lemma, which concludes the proof of Theorem 3 .

Lemma 20. Any automorphism of a germ $((M, p), \circ, e, E)$ of regular $F$ manifolds is the identity map.

Proof. Let $\psi$ be such an automorphism. Then $\psi_{*}\left(E^{i}\right)=E^{i}$ for any $i \geq 0$. From regularity, $\psi_{*}(X)=X$, i.e. $\phi_{t}^{X} \circ \psi=\psi \circ \phi_{t}^{X}$, where $\phi_{t}^{X}$ is the flow of $X$ and $X \in \mathcal{T}_{M}$ is arbitrary. Since $\psi(p)=p$, we obtain that $\psi$ is the identity map.

In the following sections we develop applications of Theorem 3 . 


\section{$5 \quad$ Frobenius metrics in canonical coordinates}

In this section we study Frobenius metrics in the coordinate system provided by Theorem 3 i). In Subsection 5.1 we express the conditions which involve the unit and Euler fields. The flatness of the metric will be treated in Subsection 5.2.

\subsection{The unit and Euler fields}

Let $M:=\mathbb{C}^{m_{1}} \times \cdots \times \mathbb{C}^{m_{n}}$. We denote by $\left(t^{i(\alpha)}\right)\left(0 \leq i \leq m_{\alpha}-1,1 \leq \alpha \leq n\right)$ the canonical coordinates on $M$ and by $\left\{\partial_{i(\alpha)}:=\frac{\bar{\partial}}{\partial t^{i(\alpha)}}\right\}$ the associated vector fields. According to Theorem $3 \mathrm{i}$ ), the multiplication

$$
\partial_{i(\alpha)} \circ \partial_{j(\beta)}=\left\{\begin{array}{l}
\partial_{(i+j)(\alpha)}, \quad \alpha=\beta, i+j \leq m_{\alpha}-1 \\
0, \quad \text { otherwise },
\end{array}\right.
$$

and the vector field

$$
E=\sum_{\alpha=1}^{n}\left(\left(t^{0(\alpha)}+a_{\alpha}\right) \partial_{0(\alpha)}+\left(t^{1(\alpha)}+1\right) \partial_{1(\alpha)}+\sum_{i=2}^{m_{\alpha}-1} t^{i(\alpha)} \partial_{i(\alpha)}\right)
$$

give $M$ the structure of an $F$-manifold, with unit field

$$
e=\sum_{\alpha=1}^{n} \partial_{0(\alpha)}
$$

Any multiplication invariant metric on $M$ is of the form

$$
g=\delta_{\alpha \beta} \eta_{(i+j)(\alpha)} d t^{i(\alpha)} \otimes d t^{j(\beta)}
$$

for some functions $\eta_{i(\alpha)}$, where $1 \leq \alpha, \beta \leq n, 0 \leq i \leq m_{\alpha}-1$ and $\eta_{i(\alpha)}=0$, for $i \geq m_{\alpha}$. (To simplify notation, in (38) and in other places we omit the summation sign).

Proposition 21. i) The coidentity $e^{b}:=g(e, \cdot)$ is closed if and only if there is a function $H$ (called a metric potential) such that $\eta_{i(\alpha)}=\partial_{i(\alpha)}(H)$ for any $i(\alpha)$.

ii) The unit field e is flat (with respect to the Levi-Civita connection $\nabla^{\mathrm{LC}}$ of $g$ ) if and only if $d\left(e^{b}\right)=0$ and $e\left(\eta_{i(\alpha)}\right)=0$, for any $i(\alpha)$.

iii) The Euler field rescales $g$ (i.e. $L_{E}(g)=D g$ for a constant $D$ ) if and only if $E\left(\eta_{i(\alpha)}\right)=(D-2) \eta_{i(\alpha)}$ for any $i(\alpha)$. 
Proof. Since $e=\sum_{\alpha=1}^{n} \partial_{0(\alpha)}$, the coidentity is given by $e^{b}=\eta_{i(\alpha)} d t^{i(\alpha)}$. It is closed if and only if it is exact, i.e. $e^{b}=d H$, for a function $H$. Claim i) follows. For claim ii), we use that $\nabla^{\mathrm{LC}}(e)=0$ if and only if $d\left(e^{b}\right)=0$ and $L_{e}(g)=0$. But

$$
\begin{aligned}
L_{e}(g)\left(\partial_{i(\alpha)}, \partial_{j(\beta)}\right) & =\delta_{\alpha \beta} e\left(\eta_{(i+j)(\alpha)}\right)-g\left(\left[e, \partial_{i(\alpha)}\right], \partial_{j(\beta)}\right)-g\left(\partial_{i(\alpha)},\left[e, \partial_{j(\beta)}\right]\right) \\
& =\delta_{\alpha \beta} e\left(\eta_{(i+j)(\alpha)}\right)
\end{aligned}
$$

where in the second line we used $e=\sum_{\alpha=1}^{n} \partial_{0(\alpha)}$ and the fact that the vector fields $\left\{\partial_{i(\alpha)}\right\}$ commute. Claim ii) follows. Claim iii) follows equally easy.

\subsection{The flatness condition}

It remains to study the flatness. For this, let us consider again Dubrovin's description of Frobenius metrics on constant (not necessarily regular) $F$ manifolds, recalled in Subsection 2.1.3. In Lemma 22 we prove that the rotation coefficient operator is determined (modulo a term $\mathcal{C}_{X}$, for $X \in \mathcal{T}_{N}$ ) by the Frobenius metric. Therefore, the generalized Darboux-Egoroff equations (5) may be written directly in terms of the metric (rather than the rotation coefficient operator). Proposition 23 below is a rewriting of Theorem 3.1 of [2]. We will apply it in order to obtain a description of Frobenius metrics on regular, globally nilpotent $F$-manifolds (see Theorem 25).

We use the notation from Subsection 2.1.3. In particular, we identify $T N$ with $T^{*} N$ using $\epsilon=\epsilon_{i j} d t^{i} \otimes d t^{j}$. We denote by $\epsilon^{-1}: T^{*} N \rightarrow T N$ this isomorphism. The induced metric on $T^{*} N$ will also be denoted by $\epsilon$. It is given by $\epsilon=\epsilon^{i j} \partial_{i} \otimes \partial_{j}$ where $\left(\epsilon^{i j}\right)$ is the inverse of $\left(\epsilon_{i j}\right)$.

Lemma 22. Let $(N, \circ, e)$ be a constant $F$-manifold, with constant multiplication invariant metric $\epsilon \in S^{2}\left(T^{*} N\right), \psi=\psi_{j} d t^{j} \in \Omega^{1}(N)$ an invertible 1 -form and $T=\epsilon^{-1}(\psi) \in \mathcal{T}_{N}$ the $\epsilon$ dual vector field. There is an $\epsilon$ symmetric endomorphism $\tilde{\gamma} \in \operatorname{End}(T N)$ which satisfies

$$
L_{\partial_{i}}(\psi)=\psi\left[\mathcal{C}_{i}, \tilde{\gamma}\right], \forall i
$$

if and only if $\epsilon(\psi, \psi)$ is constant and the endomorphism $\gamma \in \operatorname{End}(T N)$, defined by

$$
\gamma=L_{\epsilon^{-1}\left(d t^{i}\right)}(\psi) \otimes\left(\partial_{i} \circ T^{-1}\right),
$$

is $\epsilon$ symmetric. If $\epsilon(\psi, \psi)$ is constant and $\gamma$ is $\epsilon$ symmetric, then $\gamma$ satisfies (39), and, moreover, any other $\epsilon$ symmetric endomorphism $\tilde{\gamma}$, which satisfies (39), is of the form $\tilde{\gamma}=\gamma+\mathcal{C}_{X}$, for $X \in \mathcal{T}_{N}$. 
Proof. We divide the proof into several steps.

Step 1. We claim that the operator $\gamma$ defined by (40) satisfies

$$
\gamma(T)=\frac{1}{2} \epsilon^{-1}\left(d t^{i}\right)(\epsilon(\psi, \psi)) \partial_{i} \circ T^{-1}
$$

To prove (41), we use the definition of $\gamma$ and $L_{\partial_{i}}(\epsilon)=0$ :

$$
\begin{aligned}
\gamma(T) & =L_{\epsilon^{-1}\left(d t^{i}\right)}(\psi)(T) \partial_{i} \circ T^{-1}=\epsilon\left(L_{\epsilon^{-1}\left(d t^{i}\right)}(\psi), \psi\right) \partial_{i} \circ T^{-1} \\
& =\frac{1}{2} \epsilon^{-1}\left(d t^{i}\right)(\epsilon(\psi, \psi)) \partial_{i} \circ T^{-1}
\end{aligned}
$$

Step 2. We claim that any $\epsilon$ symmetric endomorphism $\tilde{\gamma}$ of $T N$ satisfies

$$
\tilde{\gamma}=\epsilon^{i k} \psi\left[\mathcal{C}_{k}, \tilde{\gamma}\right]\left(\partial_{j}\right) d t^{j} \otimes\left(\partial_{i} \circ T^{-1}\right)+\mathcal{C}_{\tilde{\gamma}(T) \circ T^{-1}}
$$

To prove (42), let $\tilde{\gamma}$ be such an endomorphism. Using that $\mathcal{C}_{i}$ and $\tilde{\gamma}$ are $\epsilon$ symmetric, we obtain

$$
\psi\left[\mathcal{C}_{i}, \tilde{\gamma}\right]\left(\partial_{j}\right)=\epsilon\left(T,\left[\mathcal{C}_{i}, \tilde{\gamma}\right]\left(\partial_{j}\right)\right)=\epsilon\left(T \circ \tilde{\gamma}\left(\partial_{j}\right)-\tilde{\gamma}(T) \circ \partial_{j}, \partial_{i}\right) .
$$

From $X=\epsilon^{i k} \epsilon\left(X, \partial_{k}\right) \partial_{i}$, for any $X \in \mathcal{T}_{N}$, and relation (43), we obtain

$$
\begin{aligned}
T \circ \tilde{\gamma}\left(\partial_{j}\right)-\tilde{\gamma}(T) \circ \partial_{j} & =\epsilon^{i k} \epsilon\left(T \circ \tilde{\gamma}\left(\partial_{j}\right)-\tilde{\gamma}(T) \circ \partial_{j}, \partial_{k}\right) \partial_{i} \\
& =\epsilon^{i k} \psi\left[\mathcal{C}_{k}, \tilde{\gamma}\right]\left(\partial_{j}\right) \partial_{i},
\end{aligned}
$$

which implies (42).

Step 3. We claim that if there is an $\epsilon$ symmetric endomorphism $\tilde{\gamma}$ of $T N$, which satisfies (39), then $\epsilon(\psi, \psi)$ is constant and the operator $\gamma$, defined by (40), is $\epsilon$ symmetric. Let $\tilde{\gamma}$ be such an endomorphism. From (39) and $L_{\partial_{i}}(\epsilon)=0$,

$$
\frac{1}{2} \partial_{i}(\epsilon(\psi, \psi))=\epsilon\left(L_{\partial_{i}}(\psi), \psi\right)=\epsilon\left(\psi\left[\mathcal{C}_{i}, \tilde{\gamma}\right], \psi\right)=-\epsilon\left(\left[\mathcal{C}_{i}, \tilde{\gamma}\right](T), T\right)=0
$$

(In the third equality we used that $\left[\mathcal{C}_{i}, \tilde{\gamma}\right] \in \operatorname{End}(T N)$ is $\epsilon$ skew-symmetric; owing to this, the 1 -form $\psi\left[\mathcal{C}_{i}, \tilde{\gamma}\right] \in \Omega^{1}(N)$ is $\epsilon$ dual to $-\left[\mathcal{C}_{i}, \tilde{\gamma}\right](T) \in \mathcal{T}_{N}$. In the fourth equality we used again that $\left[\mathcal{C}_{i}, \tilde{\gamma}\right]$ is $\epsilon$ skew-symmetric). Relation (44) shows that $\epsilon(\psi, \psi)$ is constant. Using (42) $(\tilde{\gamma}$ is $\epsilon$ symmetric), (39) and $\epsilon^{-1}\left(d t^{i}\right)=\epsilon^{i k} \partial_{k}$, we obtain

$$
\tilde{\gamma}=\epsilon^{i k} \partial_{k}\left(\psi_{j}\right) d t^{j} \otimes\left(\partial_{i} \circ T^{-1}\right)+\mathcal{C}_{\tilde{\gamma}(T) \circ T^{-1}}=\gamma+\mathcal{C}_{\tilde{\gamma}(T) \circ T^{-1}}
$$

Since $\tilde{\gamma}$ is $\epsilon$ symmetric, so is $\gamma$. Our claim follows. 
Step 4. We assume that $\epsilon(\psi, \psi)$ is constant and $\gamma$ is $\epsilon$ symmetric. We claim that $\gamma$ satisfies (39). Since $\epsilon(\psi, \psi)$ is constant, $\gamma(T)=0$ (see relation (41)). Since $\gamma$ is symmetric and $\gamma(T)=0$, relation (42) implies that

$$
\gamma=\epsilon^{i k} \psi\left[\mathcal{C}_{k}, \gamma\right]\left(\partial_{j}\right) d t^{j} \otimes\left(\partial_{i} \circ T^{-1}\right)
$$

On the other hand, from its definition (40),

$$
\gamma=\epsilon^{i k} \partial_{k}\left(\psi_{j}\right) d t^{j} \otimes\left(\partial_{i} \circ T^{-1}\right) .
$$

Combining (46) with (47) we obtain that $\partial_{k}\left(\psi_{j}\right)=\psi\left[\mathcal{C}_{k}, \gamma\right]\left(\partial_{j}\right)$, i.e. $\gamma$ satisfies (39), as claimed.

Step 5. In the hypothesis from Step 4, we claim that any other $\epsilon$ symmetric endomorphism $\tilde{\gamma}$, which satisfies (39), is equal to $\gamma+\mathcal{C}_{\tilde{\gamma}(T) \circ T^{-1}}$. Let $\tilde{\gamma}$ be such an endomorphism. Since it is $\epsilon$ symmetric, it satisfies (42). Using (39), relation (42) becomes $\tilde{\gamma}=\gamma+\mathcal{C}_{\tilde{\gamma}(T) \circ T^{-1}}$, as needed.

Proposition 23. Let $(N, \circ, e)$ be a constant F-manifold and $\epsilon \in S^{2}\left(T^{*} N\right)$ a constant, multiplication invariant metric. Let $\psi=\psi_{j} d t^{j} \in \Omega^{1}(N)$ be an invertible 1-form. Then the metric

$$
g(X, Y):=(\psi \circ \psi)(X \circ Y)
$$

is Frobenius on $(N, \circ, e)$ if and only if $\epsilon(\psi, \psi)$ is constant and the endomorphism

$$
\gamma=L_{\epsilon^{-1}\left(d t^{i}\right)}(\psi) \otimes\left(\partial_{i} \circ T^{-1}\right)
$$

is $\epsilon$ symmetric and satisfies the generalized Darboux-Egoroff equations (5). Above $T=\epsilon^{-1}(\psi) \in \mathcal{T}_{N}$ is $\epsilon$ dual to $\psi$.

Proof. From Theorem 9, $g$ is Frobenius if and only if there is an $\epsilon$ symmetric endomorphism $\tilde{\gamma} \in \operatorname{End}(T N)$ (a rotation coefficient operator), which satisfies (39) and the generalized Darboux-Egoroff equations (51). From Lemma 22, the existence of an $\epsilon$ symmetric endomorphism $\tilde{\gamma}$, which satisfies (39), is equivalent to the $\epsilon$ symmetry of $\gamma$ and to $\epsilon(\psi, \psi)$ being constant. Suppose that these equivalent conditions hold. From Lemma 22 again, $\gamma=\tilde{\gamma}-\mathcal{C}_{\tilde{\gamma}(T) \circ T^{-1}}$. Therefore, $\tilde{\gamma}$ satisfies the generalized Darboux-Egoroff equations if and only if $\gamma$ does. Our claim follows.

Remark 24. There is an alternative formula for the endomorphism $\gamma$ from Lemma 22, which is more suitable for computations. Let $c_{i j}^{k}$ and $c_{k}^{i j}$ be the structure constants, in canonical coordinates $\left(t^{i}\right)$, of the multiplications on $T N$ and $T^{*} N$. We claim that

$$
\gamma=\partial_{k}\left(\psi_{j}\right) \beta_{s} \epsilon^{i k} c_{i}^{s t} d t^{j} \otimes \partial_{t}
$$


where $\beta \in \Omega^{1}(N)$ is the inverse of $\psi$. Relation (49) is obtained from the following computation: from (40),

$$
\begin{aligned}
\gamma & =\epsilon^{i k} \partial_{k}\left(\psi_{j}\right) d t^{j} \otimes\left(\partial_{i} \circ \epsilon^{-1}(\beta)\right)=\epsilon^{i k} \partial_{k}\left(\psi_{j}\right) \beta_{s} d t^{j} \otimes\left(\partial_{i} \circ \epsilon^{-1}\left(d t^{s}\right)\right) \\
& =\partial_{k}\left(\psi_{j}\right) \beta_{s} \epsilon^{i k} \epsilon^{s f} d t^{j} \otimes\left(\partial_{i} \circ \partial_{f}\right)=\partial_{k}\left(\psi_{j}\right) \beta_{s} \epsilon^{i k} \epsilon^{s f} c_{i f}^{t} d t^{j} \otimes \partial_{t} \\
& =\partial_{k}\left(\psi_{j}\right) \beta_{s} \epsilon^{i k} c_{i}^{s t} d t^{j} \otimes \partial_{t},
\end{aligned}
$$

where we used $\epsilon^{-1}\left(d t^{s}\right)=\epsilon^{s f} \partial_{f}, T^{-1}=\epsilon^{-1}(\beta)$ and $c_{i}^{s t}=\epsilon^{s f} c_{i f}^{t}$.

We now return to the setting of regular $F$-manifolds. For simplicity, we assume that $(M, \circ, e, E)$ is globally nilpotent (and regular, of dimension $m$ ). Let $\left(t^{0}, \cdots, t^{m-1}\right)$ be the coordinate system of $M$ provided by Theorem 3 i) and $\epsilon \in S^{2}\left(T^{*} M\right)$ the (multiplication invariant) metric given by

$$
\epsilon=\epsilon_{i j} d t^{i} \otimes d t^{j}, \epsilon_{i j}=\epsilon\left(\partial_{i}, \partial_{j}\right):=\delta_{i+j, m-1} .
$$

We identify $T M$ with $T^{*} M$ using $\epsilon$. The induced multiplication on $T^{*} M$ is given by $d t^{i} \circ d t^{j}=d t^{i+j-(m-1)}$ (with the convention $d t^{s}=0$ when $s \geq m$ or $s<0)$ and $d t^{m-1}$ is the unit. A 1 -form $\psi=\psi_{j} d t^{j} \in \Omega^{1}(M)$ is invertible if and only if $\psi_{m-1}$ is non-vanishing. If $\psi$ is invertible and $\beta=\beta_{j} d t^{j}$ is its inverse, then

$$
\beta_{m-1} \psi_{m-1}=1, \quad \sum_{r+s=k} \beta_{s} \psi_{r}=0, m-1 \leq k<2(m-1) .
$$

The following theorem is our main result from this section.

Theorem 25. Let $(M, \circ, e, E)$ be a regular, globally nilpotent, $m$-dimensional $F$-manifold, with fixed constant metric $\epsilon \in S^{2}\left(T^{*} M\right)$ given by (50). Let $g$ be a multiplication invariant metric, given by

$$
g=\eta_{i+j} d t^{i} \otimes d t^{j}
$$

We fix a branch of $\left(\eta_{m-1}\right)^{1 / 2}$. by

i) There is a unique invertible 1 -form $\psi=\psi_{j} d t^{j} \in \Omega^{1}(M)$, related to $g$

$$
g(X, Y)=(\psi \circ \psi)(X \circ Y), X, Y \in T M .
$$

Its $(m-1)$-component is given by $\psi_{m-1}=\left(\eta_{m-1}\right)^{1 / 2}$ and its remaining components are determined inductively by the conditions:

$$
\sum_{s+t=(m-1)+k} \psi_{s} \psi_{t}=\eta_{k}, 0 \leq k \leq m-2 .
$$


ii) The metric $g$ is Frobenius on $(M, \circ, e)$ if and only if

$$
\epsilon(\psi, \psi)=\sum_{i+j=m-1} \psi_{i} \psi_{j}
$$

is constant and

$$
\gamma:=\sum_{j} \sum_{i \leq s} \beta_{s} \partial_{m-1-i}\left(\psi_{j}\right) \partial_{m-1+i-s} \otimes d t^{j}
$$

is $\epsilon$ symmetric and satisfies the generalized Darboux-Egoroff equations (5), where $\beta:=\beta_{j} d t^{j} \in \Omega^{1}(M)$ is the inverse of $\psi$. In particular, if $g$ is Frobenius then there is (locally) a function $H$ such that $\eta_{i}=\partial_{i}(H)$, for any $i$, and $\partial_{i}(H)$ is independent of $t^{0}$.

iii) The metric $g$ is Frobenius on $(M, \circ, e, E)$ if and only if the conditions from ii) hold and, moreover, $E\left(\eta_{i}\right)=(D-2) \eta_{i}$ for any $i$.

Proof. The proof follows from Propositions 21 and 23 and relation (49).

Example 26. We consider the setting of Theorem 25.

i) The metric $\epsilon$ itself is Frobenius with $\psi=d t^{m-1}$ and $\gamma=0$.

ii) Assume that $m=2$. The 1 -form $\psi$, its inverse $\beta$, the operator $\gamma$ and $\epsilon(\psi, \psi)$ are given by

$$
\begin{aligned}
& \psi=\frac{1}{2} \eta_{0}\left(\eta_{1}\right)^{-1 / 2} d t^{0}+\left(\eta_{1}\right)^{1 / 2} d t^{1}, \beta=-\frac{1}{2}\left(\eta_{1}\right)^{-3 / 2} \eta_{0} d t^{0}+\left(\eta_{1}\right)^{-1 / 2} d t^{1} \\
& \gamma\left(\partial_{i}\right)=\beta_{1} \partial_{1}\left(\psi_{i}\right) \partial_{0}+\left(\beta_{0} \partial_{1}\left(\psi_{i}\right)+\beta_{1} \partial_{0}\left(\psi_{i}\right)\right) \partial_{1}, 0 \leq i \leq 1 \\
& \epsilon(\psi, \psi)=2 \psi_{0} \psi_{1}=\eta_{0} .
\end{aligned}
$$

The generalized Darboux-Egoroff equations reduce to $\left[\mathcal{C}_{1}, L_{\partial_{0}}(\gamma)\right]=0$. The unit field $e$ is flat if and only if $\partial_{0}\left(\eta_{1}\right)=\partial_{1}\left(\eta_{0}\right)$ and $\eta_{i}$ are independent of $t^{0}(i=1,2)$. Suppose that $e$ is flat. Then $\eta_{0}$ is constant, the generalized Darboux-Egoroff equations are automatically satisfied and $\gamma$ is $\epsilon$ symmetric. A metric is Frobenius on $(M, \circ, e)$ if and only if it is of the form $g=\dot{f}\left(d t^{0} \otimes\right.$ $d t^{1}+d t^{1} \otimes d t^{0}$ ), where $f=f\left(t^{1}\right)$ and its derivative $\dot{f}$ (with respect to $t^{1}$ ) is non-vanishing. The metric $g$ is Frobenius on $(M, \circ, e, E)$ if, moreover, $t^{1} \ddot{f}=(D-2) \dot{f}$, for a constant $D \in \mathbb{C}$.

iii) Assume that $m=3$. The 1 -form $\psi=\psi_{j} d t^{j}$, its inverse $\beta=\beta_{j} d t^{j}$ and $\epsilon(\psi, \psi)$ are given by

$$
\begin{aligned}
& \psi=\left(\frac{1}{2} \eta_{0}\left(\eta_{2}\right)^{-1 / 2}-\frac{1}{8}\left(\eta_{1}\right)^{2}\left(\eta_{2}\right)^{-3 / 2}\right) d t^{0}+\frac{1}{2} \eta_{1}\left(\eta_{2}\right)^{-1 / 2} d t^{1}+\left(\eta_{2}\right)^{1 / 2} d t^{2} \\
& \beta=\left(-\frac{1}{2} \eta_{0}\left(\eta_{2}\right)^{-3 / 2}+\frac{3}{8}\left(\eta_{1}\right)^{2}\left(\eta_{2}\right)^{-5 / 2}\right) d t^{0}-\frac{1}{2} \eta_{1}\left(\eta_{2}\right)^{-3 / 2} d t^{1}+\left(\eta_{2}\right)^{-1 / 2} d t^{2} \\
& \epsilon(\psi, \psi)=2 \psi_{0} \psi_{2}+\left(\psi_{1}\right)^{2}=\eta_{0} .
\end{aligned}
$$


Suppose that $e$ is flat. Like in the case $m=2, \eta_{i}$ are independent of $t^{0}$ and $\partial_{i}\left(\eta_{j}\right)=\partial_{j}\left(\eta_{i}\right)$, for any $i, j$. In particular, $\eta_{0}$ is constant. The operator $\gamma$ is given by: for any $0 \leq i \leq 2$,

$$
\gamma\left(\partial_{i}\right)=\partial_{2}\left(\psi_{i}\right) \beta_{2} \partial_{0}+\left(\partial_{2}\left(\psi_{i}\right) \beta_{1}+\partial_{1}\left(\psi_{i}\right) \beta_{2}\right) \partial_{1}+\left(\beta_{0} \partial_{2}\left(\psi_{i}\right)+\beta_{1} \partial_{1}\left(\psi_{i}\right)\right) \partial_{2} .
$$

It is $\epsilon$ symmetric if and only if $\gamma_{10}=\gamma_{21}, \gamma_{00}=\gamma_{22}$ and $\gamma_{01}=\gamma_{12}$, where $\gamma\left(\partial_{i}\right)=\gamma_{j i} \partial_{j}$. Suppose that these relations are satisfied. The generalized Darboux-Egoroff equations become the highly non-trivial condition

$$
\left[\mathcal{C}_{1}, L_{\partial_{2}}(\gamma)\right]-\left[\mathcal{C}_{2}, L_{\partial_{1}}(\gamma)\right]+\left[\left[\mathcal{C}_{1}, \gamma\right],\left[\mathcal{C}_{2}, \gamma\right]\right]=0
$$

which, in terms of $\gamma_{i j}$, gives

$$
\begin{aligned}
& \partial_{2}\left(\gamma_{11}-\gamma_{00}\right)-\partial_{1}\left(\gamma_{01}\right)+\left(\gamma_{01}\right)^{2}-\left(\gamma_{11}-\gamma_{00}\right) \gamma_{02}=0 \\
& \partial_{2}\left(\gamma_{01}\right)-\partial_{1}\left(\gamma_{02}\right)-\gamma_{02} \gamma_{01}=0 \\
& \partial_{2}\left(\gamma_{02}\right)+\left(\gamma_{02}\right)^{2}=0
\end{aligned}
$$

\section{Infinitesimal symmetries in canonical coor- dinates}

Definition 27. An infinitesimal symmetry of an F-manifold $(M, \circ, e, E)$ is a vector field $X$ which preserves the multiplication and the Euler field:

$$
L_{X}(\circ)=0, \quad[X, E]=0 .
$$

Using the Jacobi identity and the general formula $L_{[X, Y]}=\left[L_{X}, L_{Y}\right]$ for the Lie derivative, we obtain that the set $\mathcal{L}$ of infinitesimal symmetries of any $F$-manifold is a subalgebra of the Lie algebra of vector fields. In this section we compute the Lie algebra $\mathcal{L}$ of germs of regular $F$-manifolds. According to Theorem 2.11 of [4], an infinitesimal symmetry of a product $F$-manifold decomposes into a product of infinitesimal symmetries of the factors. The Lie algebra $\mathcal{L}$ decomposes accordingly and, from Theorem 3 , there is no loss of generality to assume that the germ is the standard model $\left(\left(\mathbb{C}^{m}, 0\right), \circ, e, E\right)$, with coordinates $\left(t^{0}, \cdots, t^{m-1}\right)$ and $F$-manifold structure given by (2) and (3) (with no index $\alpha$ ). We begin with the following lemma.

Lemma 28. A vector field $X$ on $\left(\left(\mathbb{C}^{m}, 0\right), \circ, e, E\right)$ satisfies $L_{X}(\circ)=0$ if and only if

$$
\begin{aligned}
& {\left[\partial_{0}, X\right]=0, \quad\left[\partial_{1}, X\right] \circ \partial_{m-1}=0} \\
& {\left[\partial_{i}, X\right]=i \partial_{i-1} \circ\left[\partial_{1}, X\right], 2 \leq i \leq m-1}
\end{aligned}
$$


Proof. For any vector field $X$,

$$
L_{X}(\circ)\left(\partial_{i}, \partial_{j}\right)=\left\{\begin{array}{l}
{\left[X, \partial_{i+j}\right]-\left[X, \partial_{i}\right] \circ \partial_{j}-\partial_{i} \circ\left[X, \partial_{j}\right], \quad i+j \leq m-1,} \\
-\left[X, \partial_{i}\right] \circ \partial_{j}-\partial_{i} \circ\left[X, \partial_{j}\right] \quad i+j \geq m .
\end{array}\right.
$$

In particular,

$$
\begin{aligned}
& L_{X}(\circ)\left(\partial_{0}, \partial_{0}\right)=\left[\partial_{0}, X\right] \\
& L_{X}(\circ)\left(\partial_{1}, \partial_{j-1}\right)=\left[X, \partial_{j}\right]-\left[X, \partial_{1}\right] \circ \partial_{j-1}-\partial_{1} \circ\left[X, \partial_{j-1}\right], \quad 2 \leq j \leq m-1, \\
& L_{X}(\circ)\left(\partial_{1}, \partial_{m-1}\right)=-\left[X, \partial_{1}\right] \circ \partial_{m-1}-\partial_{1} \circ\left[X, \partial_{m-1}\right] .
\end{aligned}
$$

By induction, we obtain that the right hand side of these relations vanish if and only if the relations (55) hold. Moreover, if the relations (55) hold, then $L_{X}(\circ)\left(\partial_{i}, \partial_{j}\right)=0$, for any $i, j$ (easy check).

Proposition 29. The system of vector fields $\left\{Y_{1}, \cdots, Y_{m-1}\right\}$, defined by

$$
Y_{1}:=\left(t^{1}+1\right) \partial_{1}+\sum_{j=2}^{m-1} j t^{j} \partial_{j}, \quad Y_{k}:=\partial_{k-1} \circ Y_{1}, 2 \leq k \leq m-1,
$$

is a basis of the Lie algebra $\mathcal{L}$ of infinitesimal symmetries of the standard model $\left(\left(\mathbb{C}^{m}, 0\right), \circ, e, E\right)$ and

$$
\left[Y_{i}, Y_{j}\right]=\left\{\begin{array}{l}
(i-j) Y_{i+j-1}, \quad i+j \leq m \\
0, \quad i+j>m
\end{array}\right.
$$

Proof. It is easy to check that $Y_{1}$ satisfies the relations (55) and $\left[E, Y_{1}\right]=0$, i.e. $Y_{1}$ belongs to $\mathcal{L}$. Using that $Y_{1} \in \mathcal{L}, L_{\partial_{k}}(\circ)=0$ and that $E$ is an Euler field, we obtain: for any $k \geq 2$,

$$
\begin{aligned}
& {\left[E, Y_{k}\right]=\left[E, \partial_{k-1} \circ Y_{1}\right]=\left[E, \partial_{k-1}\right] \circ Y_{1}+\partial_{k-1} \circ\left[E, Y_{1}\right]+\partial_{k-1} \circ Y_{1}=0} \\
& L_{Y_{k}}(\circ)=L_{\partial_{k-1} \circ Y_{1}}(\circ)=\partial_{k-1} \circ L_{Y_{1}}(\circ)+Y_{1} \circ L_{\partial_{k-1}}(\circ)=0 .
\end{aligned}
$$

We proved that $Y_{k} \in \mathcal{L}$, for any $k \geq 1$. Relation (56) can be checked directly.

Consider now an arbitrary vector field $X \in \mathcal{L}$. We write it as $X=$ $f_{0} \partial_{0}+f_{1} Y_{1}+\cdots+f_{m-1} Y_{m-1}$, where $f_{k}$ are functions. We will prove that $f_{0}=0$ and $f_{k}$ are constant, for any $k \geq 1$. For any $s$ (sufficiently close to $0), \Phi_{s}^{X}$ is an automorphism of the $F$-manifold. Within the $F$-manifold, the hypersurfaces $\left\{t \mid t^{0}+a=\right.$ const $\}$ are the subspaces where the only eigenvalue of $\mathcal{U}=E$ o, namely $t^{0}+a$, is constant. As $\Phi_{s}^{X}$ is an automorphism which respects multiplication and Euler field, it does not change this eigenvalue. 
Therefore the flow of $X$ respects the hypersurfaces $\left\{t \mid t^{0}+a=\right.$ const $\}$ and we obtain that $f_{0}=0$. Now, subtracting from $X$ a suitable linear combination

of $Y_{1}, \ldots, Y_{m-1}$ (with constant coefficients), we can suppose that $f_{1}(0)=\ldots=$ $f_{m-1}(0)=0$. But then the flow $\Phi_{s}^{X}$, for any $s$, fixes the point 0 , so it is an automorphism of the germ $\left(\left(\mathbb{C}^{m}, 0\right), \circ, e, E\right)$. By Lemma $20, \Phi_{s}^{X}=$ Id. This implies $X=0$.

Remark 30. The $F$-manifold in Theorem $2 \mathrm{i}),\left(\mathbb{C}^{m}, \circ, e, E\right)$ with $\circ, e$ and $E$ given there (with no index $\alpha$ ), is regular and globally nilpotent on $\mathbb{C} \times(\mathbb{C}-$ $\{1\}) \times \mathbb{C}^{m-2}$. By Theorem 2 ii), for any two values $t_{1}, t_{2} \in \mathbb{C} \times(\mathbb{C}-\{1\}) \times$ $\mathbb{C}^{m-2}$ with $t_{1}^{0}=t_{2}^{0}$, the germs $\left(\left(\mathbb{C}^{m}, t_{1}\right), \circ, e, E\right)$ and $\left(\left(\mathbb{C}^{m}, t_{2}\right), \circ, e, E\right)$ are isomorphic, and the isomorphism is unique. The flows $\Phi_{s}^{X}$ of the infinitesimal vector fields $X$ in Proposition 30 realize these isomorphisms for nearby germs.

\section{Regular F-manifolds and meromorphic con- nections}

Let $\nabla^{0}$ be a meromorphic connection on the trivial vector bundle $V^{0}=$ $D \times \mathbb{C}^{n} \rightarrow D$ (where $D$ is a small disc around the origin in $\mathbb{C}$ ), with connection form $\Omega^{0}$ given by (12), in the standard trivialization of $V^{0}$. We assume that $B_{0}^{o} \in M_{n}(\mathbb{C})$ is regular. Let $M^{\text {can }}=M^{\text {can }}\left(B_{0}^{o}, B_{\infty}\right)$ be the parameter space of the Malgrange universal deformation $\nabla^{\text {can }}$ of $\nabla^{0}$ (see Definition 14). Recall that it is the maximal integrable submanifold of the distribution $\left.\mathcal{D}\right|_{W}$, defined by (13), passing through 0 . The tangent bundle $T M^{\text {can }}$ admits a natural multiplication $\circ_{\text {can }}$ : for any $\Gamma \in M^{\text {can }},\left(\circ_{\text {can }}\right)_{\Gamma}$, acting on $T_{\Gamma} M^{\text {can }}=\mathcal{D}_{\Gamma} \subset$ $M_{n}(\mathbb{C})$, is the multiplication of matrices (it preserves $\mathcal{D}_{\Gamma}$ ). It is clear that $o_{\text {can }}$ is associative, commutative, with unit field $\left(\operatorname{Id}_{\text {can }}\right)_{\Gamma}=\operatorname{Id}$ (the identity matrix), for any $\Gamma \in M^{\text {can }}$.

Proposition 31. i) The multiplication $\circ_{\text {can }}$ gives $M^{\text {can }}$ the structure of a (regular) F-manifold, with Euler field

$$
\left(E_{\mathrm{can}}\right)_{\Gamma}:=-\left(B_{0}\right)_{\Gamma}=-B_{0}^{o}+\Gamma-\left[B_{\infty}, \Gamma\right], \quad \Gamma \in M^{\mathrm{can}} .
$$

ii) Conversely, let $(M, \circ, e, E)$ be a regular $F$-manifold, $p \in M$, and $-B_{0}^{o}$ the representation of $\mathcal{U}_{p}: T_{p} M \rightarrow T_{p} M, \mathcal{U}_{p}(X)=X \circ E_{p}$, in a basis of $T_{p} M$. Let $B_{\infty}$ be any matrix and $M^{\text {can }}:=M^{\text {can }}\left(B_{0}^{o}, B_{\infty}\right)$. The germs $((M, p), \circ, e, E)$ and $\left(\left(M^{\mathrm{can}}, 0\right), \circ_{\mathrm{can}}, \mathrm{Id}_{\mathrm{can}}, E_{\mathrm{can}}\right)$ are isomorphic.

Proof. Let $V=M^{\text {can }} \times \mathbb{C}^{n} \rightarrow M^{\text {can }}$ be the trivial bundle. Elements of $V$ are pairs $(\Gamma, v)$ where $\Gamma \in M^{\text {can }}$ and $v \in \mathbb{C}^{n}$. We shall denote by $V_{\Gamma}=\mathbb{C}^{n}$ the 
fiber of $V$ at $\Gamma \in M^{\text {can }}$. From Proposition 12 and relation (15), $\nabla^{\text {can }}$ induces a Saito structure $\left(D, \Phi, B_{0},-B_{\infty}\right)$ on $V$, as follows: $D$ is the canonical flat connection (the constant sections of $V$ are $D$-flat); $\Phi \in \Omega^{1}\left(M^{\text {can }}\right.$, $\left.\operatorname{End}(V)\right)$ is given by $\Phi_{X}=X \in M_{n}(\mathbb{C})=\operatorname{End}\left(V_{\Gamma}\right)$, for any $X \in T_{\Gamma}\left(M^{\text {can }}\right) \subset M_{n}\left(\mathbb{C}^{n}\right)$ (i.e. for any $v \in V_{\Gamma}=\mathbb{C}^{n}, \Phi_{X}(v)=X(v)$ is the action of the matrix $X$ on the vector $v) ;\left(B_{0}\right)_{\Gamma},\left(B_{\infty}\right)_{\Gamma} \in \operatorname{End}\left(V_{\Gamma}\right)$ are given by

$$
\left(B_{0}\right)_{\Gamma}=B_{0}^{o}-\Gamma+\left[B_{\infty}, \Gamma\right],\left(B_{\infty}\right)_{\Gamma}=B_{\infty} .
$$

Let $v \in \mathbb{C}^{n}$ be a cyclic vector for $B_{0}^{o}$ and $s \in \Gamma(V)$ the associated constant section. Thus, $s: M^{\text {can }} \rightarrow V=M^{\text {can }} \times \mathbb{C}^{n}, s(\Gamma)=(\Gamma, v)$, for any $\Gamma \in M^{\text {can }}$. The map

$$
I: T M^{\text {can }} \rightarrow V, I(X):=\Phi_{X}(s)=(\Gamma, X(v)), X \in T_{\Gamma} M^{\text {can }}
$$

is an isomorphism. From the definition of $\circ_{\text {can }}$ and $E_{\text {can }}, \Phi_{X \circ_{\text {can }} Y}(s)=$ $\Phi_{X} \Phi_{Y}(s)$ and $\Phi_{E_{\text {can }}}(s)=-B_{0}(s)$, i.e. $E_{\text {can }}=-I^{-1} B_{0}(s)$. It follows that $\left(\circ_{\text {can }}, E_{\text {can }}\right)$ is induced from the Saito bundle $\left(V, D, \Phi, B_{0},-B_{\infty}\right)$, as in Proposition 11. In particular, $\left(M^{\text {can }}, \circ_{\text {can }}, \operatorname{Id}_{\text {can }}, E_{\text {can }}\right)$ is a (regular) $F$-manifold, as required. This proves claim $i)$.

For claim $i i)$, let $\mathcal{U}_{\text {can }} \in \operatorname{End}\left(T M^{\text {can }}\right)$ be defined by $\mathcal{U}_{\text {can }}(X):=X \circ_{\text {can }} E_{\text {can }}$. From Proposition [11, $\left(\mathcal{U}_{\text {can }}\right)_{0}$ is conjugated to $-\left(B_{0}\right)_{0}=-B_{0}^{o}$. Since $-B_{0}^{o}$ is the representation of $\mathcal{U}_{p}$ in a basis of $T_{p} M,\left(\mathcal{U}_{\text {can }}\right)_{0}$ and $\mathcal{U}_{p}$ belong to the same conjugacy class. We conclude with Theorem 3 .

Corollary 32. Any regular $F$-manifold $(M, \circ, e, E)$ is the parameter space of an integrable deformation of a meromorphic connection on $V^{0}=D \times \mathbb{C}^{n} \rightarrow$ $D$, in Birkhoff normal form, with a pole of Poincaré rank one in the origin.

Proof. Trivial, from Proposition 31 ii).

\section{Initial conditions for Frobenius metrics}

In this section we prove an initial condition theorem for Frobenius metrics on regular $F$-manifolds (see Theorem 34 below). Our argument relies on Theorem 3 and the theory developed in [7]. A self contained proof for the existence of a Frobenius metric with given initial condition, which avoids the technicalities of [7], will be presented in Section 9. The following remark justifies the properties of $\mathcal{V}_{p}$ from Theorem 34 .

Remark 33. If $(M, \circ, e, E, g)$ is a Frobenius manifold and $L_{E}(g)=D g$ then $\nabla^{\mathrm{LC}} E=\mathcal{V}+\frac{D}{2} \mathrm{Id}$, where $\nabla^{\mathrm{LC}}$ is the Levi-Civita connection of $g$ and $\mathcal{V}$ is the $g$ skew-symmetric part of $\nabla^{\mathrm{LC}} E$. Using $[e, E]=e$ and $\nabla^{\mathrm{LC}}(e)=0$, we obtain $\mathcal{V}(e)=\left(1-\frac{D}{2}\right) e$. 
Our main result from this section is the following.

Theorem 34. Let $(M, \circ, e, E)$ be a regular $F$-manifold and $p \in M$. Suppose that $g_{p} \in S^{2}\left(T_{p}^{*} M\right)$ and $\mathcal{V}_{p} \in \operatorname{End}\left(T_{p} M\right)$ are given, such that the following conditions are satisfied:

i) $g_{p}$ is multiplication invariant and non-degenerate;

ii) $\mathcal{V}_{p}$ is $g_{p}$ skew-symmetric and $\mathcal{V}_{p}\left(e_{p}\right)=\left(1-\frac{D}{2}\right) e_{p}$, for $D \in \mathbb{C}$.

Then $g_{p}$ can be extended to a unique Frobenius metric $g$ on the germ $((M, p), \circ, e, E)$, such that $\left.\left(\nabla^{\mathrm{LC}} E\right)\right|_{T_{p} M}=\mathcal{V}_{p}+\frac{D}{2} \mathrm{Id}$.

Proof. We consider the linear data $\left(T_{p} M, \mathcal{U}_{p}, \mathcal{V}_{p}, g_{p}\right)$ (as usual, $\mathcal{U}_{p}$ is the multiplication by $\left.E_{p}\right)$. From regularity, $e_{p}$ together with $\mathcal{U}_{p}^{k}\left(e_{p}\right)(k \geq 1)$, generate $T_{p} M$. Therefore, we can apply Theorem 4.5 of [7], with the Frobenius type structure reduced to the vector space $\left(T_{p} M, \mathcal{U}_{p}, \mathcal{V}_{p}, g_{p}\right)$ and $\tau:=e_{p}$ (see also Remark 4.6 of [7]). We obtain a germ of Frobenius manifolds $((\tilde{M}, \tilde{p}), \tilde{o}, \tilde{e}, \tilde{E}, \tilde{g})$, with $L_{\tilde{E}}(\tilde{g})=D \tilde{g}$, and an isomorphism

$$
j:\left(T_{p} M, e_{p}, \mathcal{U}_{p}, \mathcal{V}_{p}, g_{p}\right) \rightarrow\left(T_{\tilde{p}} \tilde{M}, \tilde{e}_{\tilde{p}}, \tilde{\mathcal{U}}_{\tilde{p}},\left.\left(\tilde{\nabla}^{\mathrm{LC}} \tilde{E}\right)\right|_{T_{\tilde{p}} \tilde{M}}-\frac{D}{2} \mathrm{Id}, \tilde{g}_{\tilde{p}}\right)
$$

(where $\tilde{\mathcal{U}}_{\tilde{p}}$ is the multiplication by $\tilde{E}_{\tilde{p}}$ and $\tilde{\nabla}^{\mathrm{LC}}$ is the Levi-Civita connection of $\tilde{g})$. Since $j\left(e_{p}\right)=\tilde{e}_{\tilde{p}}$ and $j \circ \mathcal{U}_{p}=\tilde{\mathcal{U}}_{\tilde{p}} \circ j$, we obtain that $j\left(E_{p}^{k}\right)=\tilde{E}_{\tilde{p}}^{k}$, for any $k \geq 0$. Since $\mathcal{U}_{p}$ and $\tilde{\mathcal{U}}_{\tilde{p}}$ are conjugated, the germs $((M, p), \circ, e, E)$ and $((\tilde{M}, \tilde{p}), \tilde{o}, \tilde{e}, \tilde{E})$ are isomorphic (from Theorem 3$)$. Let $f:((M, p), \circ, e, E) \rightarrow$ $((\tilde{M}, \tilde{p}), \tilde{o}, \tilde{e}, \tilde{E})$ be an isomorphism and $g:=f^{*} \tilde{g}$. The metric $g$ is Frobenius on $((M, p), \circ, e, E)$. Since $f_{*}(e)=\tilde{e}, f_{*}(E)=\tilde{E}$ and $f_{*}$ preserves multiplications, $f_{*}\left(E^{k}\right)=\tilde{E}^{k}$, for any $k \geq 0$. In particular, $\left(f_{*}\right)_{p}\left(E_{p}^{k}\right)=\tilde{E}_{\tilde{p}}^{k}$ and hence $\left(f_{*}\right)_{p}=j$. It follows that $\left.g\right|_{T_{p} M \times T_{p} M}=j^{*}\left(\tilde{g}_{\tilde{p}}\right)=g_{p}$, i.e. $g$ extends $g_{p}$. The Levi-Civita connections $\nabla^{\mathrm{LC}}$ and $\tilde{\nabla}^{\mathrm{LC}}$ are related by

$$
f_{*} \nabla_{X}^{\mathrm{LC}}(Y)=\tilde{\nabla}_{f_{*}(X)}^{\mathrm{LC}} f_{*}(Y), \quad X, Y \in \mathcal{T}_{M} .
$$

Applying this relation to $Y:=E$, using that $f_{*}(E)=\tilde{E},\left(f_{*}\right)_{p}=j$ and

$$
\left(\left.\left(\tilde{\nabla}^{\mathrm{LC}} \tilde{E}\right)\right|_{T_{\tilde{p}} \tilde{M}}-\frac{D}{2} \mathrm{Id}\right) \circ j=j \circ \mathcal{V}_{p}
$$

(from (58)), we obtain

$$
\nabla_{X_{p}}^{\mathrm{LC}}(E)=j^{-1} \tilde{\nabla}_{j\left(X_{p}\right)}^{\mathrm{LC}}(\tilde{E})=\mathcal{V}_{p}\left(X_{p}\right)+\frac{D}{2} X_{p}, X_{p} \in T_{p} M
$$

as required. The existence of the extension is proved.

The unicity follows also from Theorem 4.5 of [7]. More precisely, from this theorem we know that any two extensions of $\tilde{g}_{p}$, with the required properties, are related by an isomorphism of the germ $((M, p), \circ, e, E)$. But any such isomorphism is the identity map (see Lemma 201). Our claim follows. 
Remark 35. In [6] (Chapter 3) it was asked whether there exist F-manifolds which do not admit, in the neighborhood of any point, any Frobenius metric. There are F-manifolds for which the answer to this question is not known (e.g. some generically semisimple F-manifolds near points where they are not semisimple). Below we describe two sources of examples for which the answer is negative.

a) Proposition 5.32 and Remark 5.33 of 4 ] provide examples of germs $(M, 0)$ of generically semisimple F-manifolds such that $T_{0} M$ is a local algebra, but not a Frobenius algebra, so it does not allow a nondegenerate multiplication invariant metric. In Proposition 5.32 of [4 the F-manifolds are 3 dimensional, and $T_{0} M$ is as an algebra isomorphic to $\mathbb{C}\{x, y\} /\left(x^{2}, x y, y^{2}\right)$.

b) There are examples of (globally nilpotent) $F$-manifolds which do not support any Frobenius metric. Such $F$-manifolds are described in [8], Sections 2.5.2 and 2.5.3. Recall that an associative, commutative, with unit multiplication $\circ$ on the tangent bundle $T M$ of a manifold $M$ defines a (possible non-reduced) subvariety $Y$ of $T^{*} M$, the spectral cover, by the ideal $I=\left(y^{0}-1, y^{i} y^{j}-\sum_{k} a_{i j}^{k}(x) y^{k}\right) \subset \mathcal{O}_{T^{*} M}$, where $\left(x^{i}\right)$ are coordinates on $M$, with $\partial_{0}=e$ the unit field, $\left(x^{i}, y^{j}\right)$ are the induced coordinates on $T^{*} M$ and $a_{i j}^{k}$ are defined by $\partial_{i} \circ \partial_{j}=a_{i j}^{k} \partial_{k}$. The integrability condition (1) from the definition of $F$-manifolds is equivalent to $\{I, I\} \subset I$, where $\{\cdot, \cdot\}$ is the canonical Poisson bracket of $T^{*} M$ (see Theorem 2.5 of [8]). The reduced variety $Y_{\text {red }}$, defined by $\sqrt{I}$, is the support of the Higgs bundle $\left(T M, \mathcal{C}_{X}(Y)=X \circ Y\right)$ :

$Y_{\text {red }}=\cup_{x \in M}\left\{\lambda \in T_{x}^{*} M, \quad \forall X \in T_{x} M, \operatorname{ker}\left(\mathcal{C}_{X}-\lambda(X)\right.\right.$ id $\left.\left.: T_{x} M \rightarrow T_{x} M\right) \neq 0\right\}$.

If the F-manifold can be enriched to a Frobenius manifold (even without Euler field), this induces on the pull back of $T^{*} M$ to $\mathbb{C} \times M$ a (T)-structure (in the notation of [3]) respectively a holonomic $\mathcal{R}_{\mathcal{X}}$ module (in the notation of [16], where $X=M)$. This is essentially the construction of the Saito bundle from the Frobenius manifold, but without the data from the Euler field. A result of Sabbah ([16], Proposition 1.2.5) on holonomic $\mathcal{R}_{\mathcal{X}}$-modules says that the reduced variety $Y_{\text {red }}$ is Lagrangian, or, equivalently, $\{\sqrt{I}, \sqrt{I}\} \subset \sqrt{I}$. The ideals defining the spectral covers in the examples of $F$-manifolds from [8], mentioned above, do not satisfy this last condition. Thus, these $F$-manifolds do not support any Frobenius metric.

\section{Appendix: proof of Theorem 34 revised}

As promised in Section 8, we develop here an alternative argument for the existence part in Theorem 34. Consider the setting from this theorem. Let 
$B_{0}^{o}$ and $B_{\infty} \in M_{n}(\mathbb{C})$ be the matrix representations of $\mathcal{U}_{p}=\left(\mathcal{C}_{E}\right)_{p}$ and $\mathcal{V}_{p}$ in the basis $\mathcal{B}:=\left\{e_{p}, E_{p}, \cdots, E_{p}^{n-1}\right\}$ of $T_{p} M$ (where $\left.n:=\operatorname{dim}(M)\right)$ :

$$
\mathcal{U}_{p}\left(E_{p}^{i}\right)=\left(B_{0}^{o}\right)_{j i} E_{p}^{j}, \mathcal{V}_{p}\left(E_{p}^{i}\right)=\left(B_{\infty}\right)_{j i} E_{p}^{j} .
$$

For any $0 \leq i \leq n-2$,

$$
\left(B_{0}^{o}\right)_{i+1, i}=1, \quad\left(B_{0}^{o}\right)_{j i}=0, j \neq i+1 .
$$

Since $\mathcal{V}_{p}\left(e_{p}\right)=\left(1-\frac{D}{2}\right) e_{p}$

$$
\left(B_{\infty}\right)_{j 0}=\left(1-\frac{D}{2}\right) \delta_{0 j}, 0 \leq j \leq n-1 .
$$

From the skew-symmetry of $\mathcal{V}_{p}$,

$$
\left(B_{\infty}\right)_{k i} e_{p}^{b}\left(E_{p}^{k+j}\right)+\left(B_{\infty}\right)_{k j} e_{p}^{b}\left(E_{p}^{k+i}\right)=0, \quad 0 \leq i, j \leq n-1,
$$

where $e_{p}^{b}(X)=g_{p}\left(e_{p}, X\right)$, for any $X \in T_{p} M$.

Let $M^{\text {can }}:=M^{\text {can }}\left(-B_{0}^{o},-B_{\infty}\right)$, with its $F$-manifold structure provided by Proposition 31, From this proposition, we know that there is an isomorphism

$$
f:((M, p), \circ, e, E) \rightarrow\left(\left(M^{\mathrm{can}}, 0\right), \circ_{\mathrm{can}}, \mathrm{Id}_{\mathrm{can}}, E_{\mathrm{can}}\right) .
$$

Recall that the Euler field of $M^{\text {can }}$ is given by

$$
\left(E_{\mathrm{can}}\right)_{\Gamma}:=B_{0}^{o}+\Gamma+\left[B_{\infty}, \Gamma\right], \Gamma \in M^{\mathrm{can}} .
$$

In particular, $\left(E_{\text {can }}\right)_{0}=B_{0}^{o}$ and, since $f_{*}\left(E^{i}\right)=\left(E_{\text {can }}\right)^{i}$, we obtain that

$$
\left(f_{*}\right)_{p}\left(E_{p}^{i}\right)=\left(B_{0}^{o}\right)^{i}, \quad i \geq 0
$$

Let

$$
\left(g_{\text {can }}\right)_{0}: T_{0} M^{\text {can }} \times T_{0} M^{\text {can }} \rightarrow \mathbb{C}, \quad\left(g_{\text {can }}\right)_{0}:=\left(f_{*}^{-1}\right)\left(g_{p}\right)
$$

be the push-forward metric, given by

$$
\left(g_{\text {can }}\right)_{0}\left(\left(B_{0}^{o}\right)^{i},\left(B_{0}^{o}\right)^{j}\right)=g_{p}\left(E_{p}^{i}, E_{p}^{j}\right)=e_{p}^{b}\left(E_{p}^{i+j}\right), \quad 0 \leq i, j \leq n-1 .
$$

The endomorphism $\left(\mathcal{U}_{\text {can }}\right)_{0}(X)=X \circ_{\text {can }} E_{\text {can }}$ of $T_{0} M^{\text {can }}$ is the multiplication by $B_{0}^{o} \in M_{n}(\mathbb{C})$ on $T_{0} M^{\text {can }} \subset M_{n}(\mathbb{C})$. It is $\left(g_{\text {can }}\right)_{0}$ symmetric. Using the isomorphism (62), the existence part in Theorem 34 is a consequence of the following lemma. 
Lemma 36. The metric $\left(g_{\mathrm{can}}\right)_{0}$ defined by 64) admits an extension to a Frobenius metric $g_{\mathrm{can}}$ on the germ $\left(\left(M^{\mathrm{can}}, 0\right), \circ_{\mathrm{can}}, \mathrm{Id}_{\mathrm{can}}, E_{\mathrm{can}}\right)$, such that

$$
\left(D^{\mathrm{LC}} E_{\text {can }}\right)_{0}=\left(f_{*}\right)_{p} \circ \mathcal{V}_{p} \circ\left(f_{*}^{-1}\right)_{p}+\frac{D}{2} \mathrm{Id} .
$$

Above $D^{\mathrm{LC}}$ is the Levi-Civita connection of $g_{\mathrm{can}}$.

Proof. We preserve the notation from the proof of Proposition 31. Let $V=$ $M^{\text {can }} \times \mathbb{C}^{n} \rightarrow M^{\text {can }}$ be the trivial bundle over $M^{\text {can }}=M^{\text {can }}\left(-B_{0}^{o},-B_{\infty}\right)$ and $s \in \Gamma(V)$ the constant section $s(\Gamma)=\left(\Gamma, v_{0}\right)$, where $v_{0}:=(1,0, \cdots, 0) \in$ $\mathbb{C}^{n}$. We denote by $v_{1}:=(0,1,0, \cdots, 0), v_{2}:=(0,0,1,0, \cdots, 0), \ldots, v_{n-1}=$ $(0, \cdots, 0,1)$ the remaining standard vectors of $\mathbb{C}^{n}$. The $F$-manifold structure of $M^{\text {can }}$ is obtained (as explained in Proposition 11) from the Saito bundle $\left(V, D, \Phi, B_{0}, B_{\infty}\right)$ (defined as in the proof of Proposition 31, with $B_{0}^{o}$ replaced by $-B_{0}^{o}$ and $B_{\infty}$ by $-B_{\infty}$ ), by means of the isomorphism

$$
I: T M^{\mathrm{can}} \rightarrow V, I(X)=\Phi_{X}(s)=\left(\Gamma, X\left(v_{0}\right)\right), X \in T_{\Gamma} M^{\text {can }} \subset M_{n}(\mathbb{C}) .
$$

From (59), $v_{0}$ is a cyclic vector for $B_{0}^{o}$ and

$$
I_{0}: T_{0} M^{\text {can }} \rightarrow V_{0}=\mathbb{C}^{n}, I_{0}\left(\left(B_{0}^{o}\right)^{i}\right)=\left(B_{0}^{o}\right)^{i}\left(v_{0}\right)=v_{i}, \quad 0 \leq i \leq n-1 .
$$

Let $g_{0}:=\left(I_{0}^{-1}\right)^{*}\left(g_{\text {can }}\right)_{0} \in S^{2}\left(V_{0}^{*}\right)$ be the push-forward of $\left(g_{\text {can }}\right)_{0} \in S^{2}\left(T_{0}^{*} M^{\text {can }}\right)$ :

$$
g_{0}\left(v_{i}, v_{j}\right):=\left(g_{\text {can }}\right)_{0}\left(I_{0}^{-1}\left(v_{i}\right), I_{0}^{-1}\left(v_{j}\right)\right)=\left(g_{\text {can }}\right)_{0}\left(\left(B_{0}^{o}\right)^{i},\left(B_{0}^{o}\right)^{j}\right)=e_{p}^{b}\left(E_{p}^{i+j}\right) .
$$

Since $\left(\mathcal{U}_{\text {can }}\right)_{0}$ is $\left(g_{\text {can }}\right)_{0}$ symmetric (as stated before the lemma), $I_{0} \circ\left(\mathcal{U}_{\text {can }}\right)_{0} \circ$ $I_{0}^{-1}$ is $g_{0}$ symmetric. But $I_{0} \circ\left(\mathcal{U}_{\text {can }}\right)_{0} \circ I_{0}^{-1}=-\left(B_{0}\right)_{0}$ (see Proposition 11). Since $B_{0} \in \operatorname{End}(V)$ is given by

$$
\left(B_{0}\right)_{\Gamma}=-\left(B_{0}^{o}+\Gamma+\left[B_{\infty}, \Gamma\right]\right), \quad \Gamma \in M^{\text {can }},
$$

we obtain that $-\left(B_{0}\right)_{0}=B_{0}^{o}$. Therefore, $B_{0}^{o} \in M_{n}(\mathbb{C})$ is $g_{0}$ symmetric. From (61) and (68), $B_{\infty} \in M_{n}(\mathbb{C})$ is $g_{0}$ skew-symmetric. Since $B_{0}^{o}$ is $g_{0}$ symmetric and $B_{\infty}$ is $g_{0}$ skew-symmetric, $\left(B_{0}\right)_{\Gamma}$ is $g_{0}$ symmetric when $\Gamma$ is so.

Let $M_{n}^{\text {sym }}(\mathbb{C})$ be the manifold of $g_{0}$ symmetric matrices. We claim that $\left(M^{\text {can }}, 0\right) \subset\left(M_{n}^{\text {sym }}(\mathbb{C}), 0\right)$. For this, we use the above observation (namely, $\left(B_{0}\right)_{\Gamma} \in M_{n}^{\text {sym }}(\mathbb{C})$ when $\Gamma \in M_{n}^{\text {sym }}(\mathbb{C})$ ) and the following general fact (which can be easily checked): if $\mathcal{D}$ is an integrable distribution on a manifold $M$, $N$ is a submanifold of $M$ such that $\left.\mathcal{D}\right|_{N} \subset T N$ and $I^{\max }$ is the maximal integrable submanifold of $\mathcal{D}$, which contains $p \in N$, then there is a neighborhood $U$ of $p$ in $M$, such that $I^{\max } \cap U \subset N \cap U$. Applying this fact to $M:=W$ 
(a small open neighborhood of $\left.0 \in M_{n}(\mathbb{C})\right), N:=M_{n}^{\text {sym }}(\mathbb{C}) \cap W$ and the distribution $\left.\mathcal{D}\right|_{W}$ whose maximal integrable submanifold is $M^{\text {can }}$ (and whose fiber at $\Gamma \in W$ is the vector space of polynomials in $\left(B_{0}\right)_{\Gamma}$, with $\left(B_{0}\right)_{\Gamma}$ as in $(69)$ ), we obtain $\left(M^{\text {can }}, 0\right) \subset\left(M_{n}^{\text {sym }}(\mathbb{C}), 0\right)$, as needed.

Let $g_{V} \in S^{2}\left(V^{*}\right)$ be the constant extension of $g_{0}$ to $V$. It follows that $\left(V, D, \Phi, B_{0}, B_{\infty}, g_{V}\right)$ is a Saito bundle with metric (see Definition 10). The section $s$ is primitive homogeneous, with $B_{\infty}(s)=\left(1-\frac{D}{2}\right) s$ (we use (60); recall that $s$ is the constant section of $V$, determined by $\left.v_{0}=(1,0, \cdots, 0) \in \mathbb{C}^{m}\right)$. The metric $g_{\text {can }}(X, Y):=g_{V}(I(X), I(Y))$ extends $\left(g_{\text {can }}\right)_{0}$ (from (68) $)$. From Subsection 2.2, $g_{\text {can }}$ is a Frobenius metric on $\left(\left(M^{\text {can }}, 0\right), \circ_{\text {can }}, \operatorname{Id}_{\text {can }}, E_{\text {can }}\right)$ and

$$
D^{\mathrm{LC}} E_{\text {can }}=I^{-1} B_{\infty} I+\frac{D}{2} \mathrm{Id} .
$$

In order to conclude the proof, we need to check that

$$
I_{0}^{-1} B_{\infty} I_{0}=\left(f_{*}\right)_{p} \circ \mathcal{V}_{p} \circ\left(f_{*}^{-1}\right)_{p} .
$$

From (67), the left hand side of (70), applied to $\left(B_{0}^{o}\right)^{i}$ (with $0 \leq i \leq n-1$ ), is given by

$$
\left(I_{0}^{-1} B_{\infty} I_{0}\right)\left(B_{0}^{o}\right)^{i}=I_{0}^{-1} B_{\infty}\left(v_{i}\right)=\left(B_{\infty}\right)_{j i}\left(B_{0}^{o}\right)^{j} .
$$

From (63), the right hand side of (170), applied to $\left(B_{0}^{o}\right)^{i}$, is given by

$$
\left(\left(f_{*}\right)_{p} \circ \mathcal{V}_{p} \circ\left(f_{*}^{-1}\right)_{p}\right)\left(B_{0}^{o}\right)^{i}=\left(f_{*}\right)_{p} \mathcal{V}_{p}\left(E_{p}^{i}\right)=\left(B_{\infty}\right)_{j i}\left(f_{*}\right)_{p}\left(E_{p}^{j}\right)=\left(B_{\infty}\right)_{j i}\left(B_{0}^{o}\right)^{j}
$$

Relation (70) follows.

\section{References}

[1] L. David, I. Strachan: Dubrovin's duality for F-manifolds with eventual identities, Adv. Math (5) vol. 266, (2011), p. 4031-4060.

[2] B. Dubrovin: Geometry of 2D topological field theory, arxiv: hep-th/9407018, Springer LNM, 1620 (1996), p. 120-348.

[3] C. Hertling: $t t^{*}$-geometry, Frobenius manifolds, their connections, and the construction for singularities, J. Reine Angew. Math. 555 (2003), p. $77-161$.

[4] C. Hertling: Frobenius manifolds and moduli spaces for singularities, Cambridge University Press, (2002). 
[5] C. Hertling, L. Hoevenaars, H. Posthuma: Frobenius manifolds, projective special geometry and Hitchin systems, J. reine angew. Math. 649 (2010), p. 117-165.

[6] C. Hertling and Y. I. Manin: Weak Frobenius Manifolds, Int. Math. Res. Notices 6 (1999), p. 277-286.

[7] C. Hertling, Y. I. Manin: Unfoldings of meromorphic connections and a construction of Frobenius manifold, in Frobenius manifolds, quantum cohomology and singularities (eds. C. Hertling and M. Marcolli), ViewegTeubner, (2004), p. 113-144.

[8] C. Hertling, Y. I. Manin, C. Teleman: An Update on Semisimple Quantum Cohomology and F-manifolds, Proc. Steklov Inst. Math., vol. 264 (2009), p. 62-69.

[9] P. Lorenzoni, M. Pedroni, A. Raimondo: F-manifolds and integrable systems of hydro-dynamic type, Arch. Math. (Brno) 47, no. 3 (2011), p. 163-180.

[10] B. Malgrange: Deformations de systemes differentielles et microdifferentielles, Seminaire E.N.S. Mathematique et Physique, Progress in Math. vol. 37, Birkhäuser, Basel, Boston, p. 351-379.

[11] B. Malgrange: Deformations of differential systems, II, J. Ramanujan Math. Soc. 1 (1986), p. 3-15.

[12] Y. I. Manin: Frobenius manifolds, Quantum Cohomology and Moduli Spaces, A.M.S. Colloquim Publications, vol. 47, 1999.

[13] Y. I. Manin, S. A Merkulov: Semisimple Frobenius (super)-manifolds and quantum cohomology on $\mathbb{C} P^{n}$, Topol. Methods Nonlinear Analysis, 9 (1997), p. 107-161.

[14] S. Merkulov: Operads, deformation theory and F-manifolds, Aspects of Mathematics, vol. 36 (2004), p. 213-251.

[15] C. Sabbah: Isomonodromic deformations and Frobenius manifolds; An Introduction, Springer (2008).

[16] C. Sabbah: Polarizable twistor D-modules, Astérisque 300, Société Mathématique de France (2005).

[17] S. Sternberg: Lectures on Differential Geometry, AMS Chelsea Publishing, 1964. 
[18] I. A. B. Strachan: Frobenius manifolds: natural submanifolds and induced bi-hamiltonian structures, Diff. Geom. Applic. 20 (1) (2004), p. 67-99.

Liana David: Institute of Mathematics 'Simion Stoilow' of the Romanian Academy, Research Unit 4, Calea Grivitei nr. 21, Bucharest, Romania; liana.david@imar.ro

Claus Hertling: Lehrstuhl für Mathematik VI, Institut für Mathematik, Universität Mannheim, A5, 6, 68131, Mannheim, Germany; hertling@math.unimannheim.de 